DRAFT VERSion JANUARY 17, 2021

Preprint typeset using $\mathrm{LAT}_{\mathrm{E}} \mathrm{X}$ style emulateapj v. 5/2/11

\title{
LITHIUM ABUNDANCES IN GLOBULAR CLUSTER GIANTS: NGC 6218 (M12) AND NGC 5904 (M5) ${ }^{1}$
}

\author{
Valentina D’Orazi ${ }^{2,3}$, George C. Angelou ${ }^{3}$, Raffaele G. Gratton ${ }^{4}$, John C. Lattanzio ${ }^{3}$, Angela Bragaglia $^{5}$, \\ Eugenio Carretta ${ }^{5}$, Sara Lucatello ${ }^{4}$ and Yazan Momany ${ }^{4,6}$ \\ Draft version January 17, 2021
}

\begin{abstract}
Convergent lines of evidence suggest that globular clusters host multiple stellar populations. It appears that they experience at least two episodes of star formation whereby a fraction of firstgeneration stars contribute astrated ejecta to form the second generation(s). To identify the polluting progenitors we require distinguishing chemical signatures such as that provided by lithium. Theoretical models predict that lithium can be synthesised in AGB stars, whereas no net Li production is expected from other candidates. It has been shown that in order to reproduce the abundance pattern found in $\mathrm{M} 4$, Li production must occur within the polluters, favouring the AGB scenario. Here we present $\mathrm{Li}$ and $\mathrm{Al}$ abundances for a large sample of RGB stars in M12 and M5. These clusters have a very similar metallicity, whilst demonstrating differences in several cluster properties. Our results indicate that the first-generation and second-generation stars share the same Li content in M12; we recover an abundance pattern similar to that observed in M4. In M5 we find a higher degree of complexity and a simple dilution model fails in reproducing the majority of the stellar population. In both clusters we require Li production across the different stellar generations, but production seems to have occurred to different extents. We suggest that such a difference might be related to the cluster mass with the Li production being more efficient in less-massive clusters. This is the first time a statistically significant correlation between the Li spread within a GC and its luminosity has been demonstrated. Finally, although Li-producing polluters are required to account for the observed pattern, other mechanisms, such as MS depletion, might have played a role in contributing to the Li internal variation, though at relatively low level.
\end{abstract}

Subject headings: globular clusters: individual (NGC 6218, NGC 5904) - stars: abundances - stars: Population II

\section{INTRODUCTION}

No longer considered simple stellar populations, globular clusters (GCs) are at the crux of several unresolved problems in stellar astrophysics. Accurate photometric surveys have revealed that some clusters display multiple evolutionary sequences, from the main-sequence (MS), to the sub-giant and red-giant branches (SGB and RGB, see e.g., Bedin et al. 2004; Anderson et al. 2009; Piotto et al. 2012; Milone et al. 2012). The simplest interpretation is that there are (at least) two stellar generations present, slightly separated in age and characterised by a different chemical composition (see, however, Bastian et al. 2013 for a different view). Although this result is now clear through clever use of multifilter photometry, the seeds for the paradigm shift were planted by high-resolution spectroscopy and detailed abundance determinations across a large sample of GCs (e.g., Kraft 1994; Gratton et al. 2004; Carretta et al. 2009a, 2009b, 2011, 2013; Marino et al. 2008, 2011;

\footnotetext{
valentina.dorazi@mq.edu.au

${ }^{1}$ Based on observations taken with ESO telescopes under program 087.D-0276(A)

2 Department of Physics and Astronomy, Macquarie University, Sydney, NSW 2109, Australia.

${ }^{3}$ Monash Centre for Astrophysics, School of Mathematical Sciences, Monash University, Melbourne, VIC 3800, Australia.

${ }^{4}$ INAF - Osservatorio Astronomico di Padova, Vicolo dell'Osservatorio 5, 35122, Padova, Italy

${ }^{5}$ INAF - Osservatorio Astronomico di Bologna, via Ranzani 1, 40127, Bologna, Italy

${ }^{6}$ European Southern Observatory, Alonso de Cordova 3107, Vitacura, Santiago, Chile
}

Johnson \& Pilachowski 2010).

In contrast to field stars and open clusters (e.g., Gratton et al. 2000; de Silva et al. 2009; Bragaglia et al. 2012), archetypal GCs exhibit internal variations in elements affected by proton-capture processes (hereafter pcapture elements, such as e.g., C, N, O, F, Na, Mg, Al), but remain homogeneous in iron-peak, heavy $\alpha(\mathrm{Ca}, \mathrm{Ti})$, and neutron-capture elements (e.g., James et al. 2004; Smith 2008; D'Orazi et al. 2010a) .7

The burning patterns give rise to the well known light-element anticorrelations (C-N, O-Na, Mg-Al), with the Na-O anticorrelation suggested as the GC's defining feature (Carretta et al. 2010). The fact that these chemical peculiarities are detected also in unevolved or scarcely evolved GC stars Gratton et al. 2001; Ramírez \& Cohen 2002) implies that a fraction of first-generation (FG) stars have simultaneously activated $\mathrm{CNO}, \mathrm{NeNa}$, and (possibly) $\mathrm{MgAl}$ cycles in their interiors in order to deplete $\mathrm{C}, \mathrm{O}$ and $\mathrm{Mg}$ and enhance $\mathrm{N}, \mathrm{Na}$ and $\mathrm{Al}$, respectively. From the ejecta of these progenitors, the second-generation (SG) stars were born and currently account for the majority of the stellar population in GCs (about 2/3, see Carretta et al. 2010). Unfortunately, the nature of the progenitors responsible for the internal chemical enrichment remains unclear. Intermediate-mass asymptotic giant branch stars (IM-

7 This picture is further complicated by the presence in the large GC family of some peculiar cases (e.g., $\omega$ Centauri, M 22, NGC 1851, M15, Terzan 5, NGC 2419), where variations in metallicity and/or heavy-elements are detected (though at a different extent). 
AGB, Cottrell \& Da Costa 1981; Ventura et al. 2001) and fast rotating massive stars (FRMS, Decressin et al. 2007) remain the prime candidates, although neither offer a satisfactory explanation (we refer to Gratton et al. 2012 for an updated and comprehensive review on the multiple population framework).

Besides the imprinted abundance patterns in GCs, lighter nuclei such as ${ }^{7} \mathrm{Li}, \mathrm{C}$ and $\mathrm{N}$ display evidence of in situ processing; their abundances change as a function of RGB luminosity (beyond the extent of the first-dredge up, FDU). This is not predicted by standard stellar theory and is one example of the need for 'extra mixing' in numerical models.

Surveys of $\mathrm{C}$ and $\mathrm{N}$ in GCs have been used extensively to study the RGB extra-mixing problem (Denissenkov \& VandenBerg 2003; Angelou et al. 2011, 2012) but the recent availability of Li provides a complementary and very powerful diagnostic. By virtue of its fragility, $\mathrm{Li}$ is a sensitive probe of mixing in stars. It is produced during $\mathrm{H}$ burning when ${ }^{7} \mathrm{Be}$ captures an electron as part of the $p p$ II chain. At these temperatures ( $\mathrm{T}$ $\gtrsim 2 \mathrm{MK}$ ), it is also highly favourable for ${ }^{7} \mathrm{Li}$ to subsequently capture a proton to produce two ${ }^{4} \mathrm{He}$ nuclei. As first pointed out by Cameron \& Fowler (1971), efficient mixing in the burning region can transport the material rich in ${ }^{7}$ Be to cooler temperatures where a further proton capture is avoided. This is the so called "CameronFowler ${ }^{7} \mathrm{Be}$ transport mechanism". Conversely, ${ }^{7} \mathrm{Li}$ is easily destroyed whenever material is transported from the cool surface to the interior of the star. The surface $\mathrm{Li}$ abundance serves as a key indicator for either of these internal processes.

Lithium finds itself at the centre of another long standing discrepancy, that is its abundance, as measured in Population II dwarfs, is factors of 2-3 lower than that predicted by Big Bang nucleosynthesis (e.g., Cyburt et al. 2008). Currently, the favoured explanation for this inconsistency is that the stars themselves are responsible for the depletion via a different kind of process driven by a different mechanism (such as atomic diffusion, i.e., the transport of chemicals due to temperature, pressure and abundance gradients; see e.g., Richard et al. 2005). To date, this cosmological problem has served as the prime motivation for obtaining $\mathrm{Li}$ abundances in $\mathrm{GC}$ dwarfs (Pasquini et al. 2005; Bonifacio et al. 2007; Lind et al. 2009; González Hernández et al. 2009; Mucciarelli et al. 2011, 2012 and references therein).

D’Orazi \& Marino (2010, hereafter DM10) examined the $\mathrm{Li}$ abundances in the mildly metal-poor $\mathrm{GC}$ NGC 6121 (M4, $[\mathrm{Fe} / \mathrm{H}]=-1.16$, Harris 1996, updated in 2010), but focussed on giant stars (104 RGB stars with 32 targets located below the bump luminosity). The shift in scientific motivation encouraged a shift in target selection: rather than investigate the Spite Plateau (Spite \& Spite 1982) and hence un-evolved stars, the purpose of that study was twofold:

* Constrain the nature of the first (polluting) generation of stars in the cluster. At the high temperatures at which the $\mathrm{CNO}$ occurs $(\mathrm{T} \gtrsim 20 \mathrm{MK})$ it is expected that $\mathrm{Li}$ is completely destroyed ( $\mathrm{T}_{\text {burning }} \approx 2.5 \mathrm{MK}$ ). Thus, along with displaying the $\mathrm{O}-\mathrm{Na}, \mathrm{C}-\mathrm{N}$, and possibly $\mathrm{Mg}-\mathrm{Al}$ anticorrelations, the multiple population scenario predicts that $\mathrm{Li}$ and $\mathrm{O} / \mathrm{C} / \mathrm{Mg}$ should be positively correlated, while $\mathrm{Li}$ and $\mathrm{Na} / \mathrm{N} / \mathrm{Al}$ anticorrelated (if no $\mathrm{Li}$ is produced by the polluters). The FG stars should be Li rich, whereas stars formed from the ejecta processed at extremely high temperatures should be Li poor.

* Under the assumption that there is no Li production within the polluters, shed light on the dilution process within this $\mathrm{GC}$ and try to determine the amount of pristine (and of polluted) material present in each star.

For both these goals, any stars not yet experiencing extra mixing are suitable targets. Although Li is depleted by a factor of $\approx 20$ during FDU, the abundance change is simply considered a zero-point offset because FG and SG stars are affected in the same way 8 . Thus, DM10 could afford to turn their attention to brighter targets, provided the stars were situated below the bump in the luminosity function of the RGB (LF bump). Their main finding was the lack of a Li-Na anticorrelation in M4 with the FG (Na poor, $\mathrm{A}(\mathrm{Li})=1.34 \pm 0.04$ ) and $\mathrm{SG}$ (Na rich, $\mathrm{A}(\mathrm{Li})=1.38 \pm 0.04)$ sharing the same Li abundance. The implications are that $\mathrm{Li}$ has been produced between the different stellar generations and, crucially, this abundance pattern is not an outcome of dilution processes with primordial material. This striking result requires the progenitors to produce Li. An outcome which is not currently predicted by massive star evolution (Decressin et al. 2007) nor massive binaries (de Mink et al. 2009). DM10 therefore provided, for the first time, strong observational evidence that IM-AGB stars seem to play a significant role in the internal enrichment of GCs (at least in this system).

In this study we expand upon previous results and present Li abundances in the GCs NGC 6218 (M12) and NGC 5904 (M5). Our approach, which focuses on stars brighter than the turn-off, allows us to target more distant systems, whereas previous studies were forced to analyse the near-by GCs (M4, NGC 6397 and 47 Tuc). M12 and M5 were specifically chosen to improve our understanding of Li across the GC mass and metallicity distributions. Both clusters are similar in metallicity (and they are similar to M4) but they differ significantly in mass. We note that in NGC 6218, Carretta et al. (2007) detected that stars brighter than the LF bump possess statistically higher $\mathrm{Na}$ than those below. This is an expected result from the presence of two populations with distinct He abundances and hence different bump luminosities (Salaris et al. 2006).

This work is part of a long-term project aimed at determining homogeneous Li abundances at all RGB luminosities (hundreds of stars) in a large number of GCs.

\footnotetext{
8 Models in an upcoming paper (Angelou et al. 2014) demonstrate that such an assumption is plausible. Calculations of FG stars $\left(\mathrm{M}=0.80 \mathrm{M}_{\odot}, \mathrm{Y}=0.24\right)$ and an extreme population $(\mathrm{M}=0.80$ $\mathrm{M}_{\odot}$ and $\left.\mathrm{Y}=0.40\right)$ exhibit a a difference in $\mathrm{A}(\mathrm{Li}) \sim 0.1$ dex after FDU, on account of a slightly deeper penetration of the convective envelope. We expect that if a star is still visible on the giant branch and has such an extreme helium abundance it must necessarily be less massive $\left(\mathrm{M} \lesssim 0.65 \mathrm{M}_{\odot}\right)$. In such a star the difference in $\mathrm{A}(\mathrm{Li})$ is smaller than 0.1 dex after FDU. Even by selecting an unreasonably extreme mass and composition the models predict a difference in Li within the observational uncertainty.
} 
Clusters covering a range in mass, metallicity, HB morphology and shape/extent of the Na-O anticorrelation are required to probe the relationship between the $\mathrm{Li}$ abundance and the GC global parameters. Besides the scientific motivation addressed in this study, such a data set will also provide stringent constraints on stellar evolution and mixing processes in stars.

The paper is organised as follows: in Section 2 we provide information on sample, data reduction and abundance analysis; our results are presented and discussed in Section 3. A summary of our findings concludes the manuscript (Section 4).

\section{OBSERVATIONS, DATA REDUCTION AND ANALYSIS}

We utilised the multi-object FLAMES@VLT facility (Pasquini et al. 2002) to collect intermediate-resolution spectra of RGB stars, both below and above the bump luminosity, in our target clusters (Program: 087.2-0276(A), PI: VD). Employing the HR15N setup (6470-6790 A), our wavelength coverage included the $\mathrm{Li}$ I doublet at $6707.78 \AA$ with a nominal resolution of $\mathrm{R}=17,000$. We observed a total of 72 stars in NGC 6218 and 113 stars in NGC 5904 using this configuration. The sample was selected from the photometric catalogues provided by Momany et al. (private communication). We imposed that targets lack companions within 3 " or with companions but not closer than 2" and fainter than 2 magnitudes. We refer to Momany et al. (2003, 2004) for details about photometric data reduction and analysis. The colourmagnitude diagrams for both GCs are shown in Fig. 1. with target stars emboldened. The spectroscopic data reduction was performed by the ESO personnel through the dedicated software that produces extracted, bias subtracted, flat-field corrected and wavelength calibrated spectra. In addition, continuum normalisation, radial velocity computation, shift to rest-frame and combination of multiple exposures were all carried out within IRAFI. The typical S/N ratios (per pixel) of our target stars range from 60 to 150 at $6700 \AA$.

From the original sample, we discarded 4 stars in NGC 6218 and 6 stars in NGC 5904 as their radial velocities were more than $3 \sigma$ away from the respective cluster mean. We derived heliocentric radial velocities of $\mathrm{v}_{\text {rad }}=-43.31 \pm 0.41 \mathrm{~km} \mathrm{~s}^{-1}$ (r.m.s $=3.35 \mathrm{~km} \mathrm{~s}^{-1}, 68$ stars for NGC 6218) and $\mathrm{v}_{\text {rad }}=53.05 \pm 0.51 \mathrm{~km} \mathrm{~s}^{-1}$ (r.m.s $=5.22 \mathrm{~km} \mathrm{~s}^{-1}, 107$ stars NGC 5904), which are in reasonable agreement, in view of the zero point uncertainties, with values published by Harris (1996), that is $\mathrm{v}_{\text {rad }}=-41.4 \pm 0.2 \mathrm{~km} \mathrm{~s}^{-1}$ and $\mathrm{v}_{\text {rad }}=53.2 \pm 0.4 \mathrm{~km} \mathrm{~s}^{-1}$.

The stellar atmospheric parameters for our sample stars were derived in the following way. We first calculated initial $T_{\text {eff }}$ values from $(V-K)$ colours (with $V$ from our photometry and 2MASS $K$ magnitudes, Skrutskie et al. 2006) and the calibration by Alonso et al. (1999); metallicity and reddening were retrieved from the Harris' catalogue, that is $[\mathrm{Fe} / \mathrm{H}]=$ -1.33 and $\mathrm{E}(B-V)=0.19$ for NGC 6218 , and $[\mathrm{Fe} / \mathrm{H}]$ $=-1.29$ and $\mathrm{E}(B-V)=0.03$ for NGC 5904. The reddening values were converted to $\mathrm{E}(V-K)$ via the rela-

\footnotetext{
${ }^{9}$ IRAF is the Image Reduction and Analysis Facility, a general purpose software system for the reduction and analysis of astronomical data. IRAF is written and supported by National Optical Astronomy Observatories (NOAO) in Tucson, Arizona.
}

tionship by Cardelli et al. (1989), i.e., $\mathrm{E}(V-K)=2.75 \times$ $\mathrm{E}(B-V)$. Our final adopted temperatures come from a relationship between those $T_{\text {eff }}$ values and $V$ magnitudes, following the same approach as in Carretta et al., (2006; 2009a). To derive this relation, we used only "well-behaved" stars in each cluster, that is stars with magnitudes in both visual and infrared filters (keeping only high-quality flagged 2MASS photometry), and lying on the RGB. Comparing the stars in common between the present study and Carretta et al. (2007, 2009a) we obtained a difference (in the sense ours minus Carretta) of $\Delta T_{\text {eff }}=-52 \pm 8 \mathrm{~K}$ and $\Delta T_{\text {eff }}=5 \pm 2$ for NGC 6218 and NGC 5904, respectively. The larger difference for NGC 6218 is likely related to the different photometric catalogue adopted by Carretta et al. (2007). Surface gravities $(\log g)$ were then derived by assuming our final $T_{\text {eff }}$ values, distance moduli of $(m-M)_{V}=14.04$ and $(m-M)_{V}=14.46$ (Harris 1996), a bolometric solar magnitude of $\mathrm{M}_{b o l, \odot}=4.75$, and masses of $0.85 \mathrm{M}_{\odot}$, with the standard formula:

$$
\log \frac{g}{g_{\odot}}=\log \frac{M}{M_{\odot}}-\log \frac{L}{L_{\odot}}+4 \log \frac{T_{\text {eff }}}{T_{\text {eff }, \odot}}
$$

Finally, microturbulence velocities $(\xi)$ were computed from the relationship by Gratton et al. (1996): $\xi=$ $2.22-0.322 \times \log g$, while the input metallicity was taken from Harris (1996).

The Li abundances were inferred via spectral synthesis with the driver synth in MOOG (Sneden 1973, 2013 version) and stellar atmospheres by Castelli \& Kurucz (2004), with $\alpha$-enhancement $(+0.4$ dex) and no overshooting 10. We investigated the impact of this choice by deriving Li abundances for all our sample stars using three different sets of atmospheric models, namely the Kurucz (1993) grid with and without overshooting and the Castelli \& Kurucz (2004) with solar-scaled composition (i.e., no $\alpha$-enhancement). The differences in the derived Li abundances are always smaller than 0.05 dex and can be safely considered as negligible. Adopting the same line lists as in DM10 (see D'Orazi et al. 2010b and DM10 for details on atomic parameters), we computed a grid of synthetic spectra for each star by varying the Li abundances until the best match between observed and synthetic profiles was attained. The synthetic spectra were calculated covering a wavelength range from $6695 \AA$ to $6722 \AA$, exploiting the strong Ca I line at $6717.69 \AA$ to evaluate the spectral broadening (which was assumed to be Gaussian). We were able to determine the Li abundances for 63 stars for NGC 6218 and 99 stars for NGC 5904 , because of the occurrence of cosmic rays and/or due to lower $\mathrm{S} / \mathrm{N}$ ratios that hampered measurements in 5 and 8 stars, respectively for each GC.

The spectral coverage guaranteed by the HR15N grating, although not capturing any suitable features for $\mathrm{Na}$ and $\mathrm{O}$ abundance determinations, allowed us to investigate the $\mathrm{Al}$ content by synthesising the $\mathrm{Al}$ I doublet at $6696 \AA$ and $6698 \AA$. The adopted atomic parameters for those lines were: $\log g f=-1.35$ and $\log g f=-1.65$, for the $6696 \AA$ and $6698 \AA$ features, respectively. In NGC 6218 we could obtain $\mathrm{Al}$ abundances for a sample of 54 out of 63 stars, whereas in NGC 5904 we analysed 93

10 Available at http://kurucz.harvard.edu/grids.html 

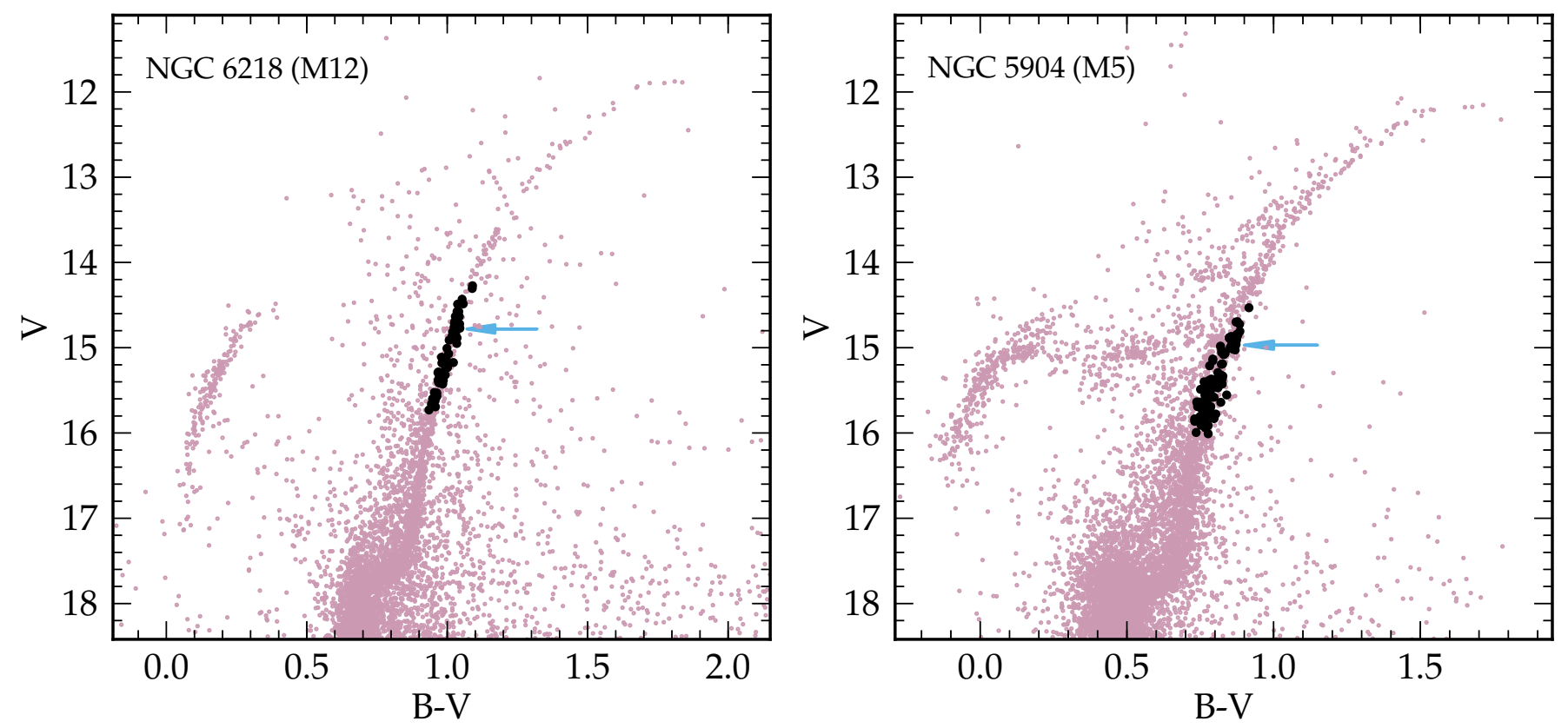

Figure 1. The colour-magnitude diagrams for the GCs M12 and M5 (left and right-hand panels, respectively). Stars targeted during this survey are emboldened. The arrows indicate the location of the LF bump from Nataf et al. (2013).

Table 1

Sensitivities of abundances to atmospheric parameters for star \#40129.

\begin{tabular}{lccr}
\hline \hline Species & $T_{\text {eff }}+100$ & $\log g+0.2$ & $\xi+0.1$ \\
\hline$\Delta \mathrm{A}(\mathrm{Li})$ & 0.10 & 0.02 & 0.00 \\
$\Delta[\mathrm{Al} / \mathrm{Fe}]$ & 0.07 & 0.06 & 0.02 \\
\hline \hline
\end{tabular}

stars (out of 99), with 61 detections and 32 upper limits. Examples of spectral synthesis for $\mathrm{Li}$ and $\mathrm{Al}$ are given in Figures 2 and 3 for stars in both clusters.

\subsection{Error budget}

Two kind of uncertainties affect our derived abundances, that is internal (star-to-star) and systematic (cluster) errors. The main aim of our paper is to search for (possible) evidence of spreads in $\mathrm{Li}$ and $\mathrm{Al}$ abundances, thus we focus on the first source of errors. The internal errors are mainly related to $(i)$ the best-fit determination of synthetic spectra with observed spectra (which is in the range $0.07-0.10$ for our target stars and reflect uncertainties in the continuum placement and $\mathrm{S} / \mathrm{N}$ ratios of the spectra) and (ii) to the atmospheric parameters, i.e., $T_{\text {eff }} \log g$, and microturbulence velocities $\xi$ (the adopted metallicity $[\mathrm{A} / \mathrm{H}]$ in the model atmosphere has a negligible impact). In order to assess the contribution related to the stellar parameters we first need to ascertain the sensitivities of our species to changes in atmospheric quantities (the partial derivatives in Equation 11). To do this we proceeded in the standard way, that is by varying one parameter at the time and inspecting the corresponding change in the resulting abundance (see Table 1 where sensitivities are reported for one sample star with median $T_{\text {eff }}$ ). The following step is to evaluate the actual error in atmospheric parameters (i.e., $\left.\sigma_{T_{\text {eff }}}, \sigma_{\text {logg }}, \sigma_{\xi}\right)$. The $\sigma_{T_{\text {eff }}}$ can be estimated from the error on the slope of the relation between initial $T_{\text {eff }}$ values (from $(V-K)$ colours) and $V$ magnitudes, which result in $18 \mathrm{~K}$ for both GCs. Errors in $\xi$ instead come from the scatter around the relationship of $\xi$ vs $\log g$ by Gratton et al. (1996, that is $\left.0.2 \mathrm{~km} \mathrm{~s}^{-1}\right)$. Finally, the $\sigma_{\text {logg }}$ contains different terms due to the uncertainties in stellar masses (which is, however, less than $\approx 10 \%$ of the mass), errors due to luminosity (in turn related to magnitudes, distance moduli and bolometric corrections) and those in temperatures. All these contributions are anyway significantly small and results in internal errors in $\log g$ values less than 0.05 dex.

The total internal error on a given species is then calculated by summing in quadrature all the different contributions, i.e.:

$$
\sigma=\sqrt{\sigma_{\text {best }}^{2}+\left(\frac{\partial \log (\epsilon)}{\partial T_{\text {eff }}}\right)^{2} \sigma_{T_{\text {eff }}}^{2}+\left(\frac{\partial \log (\epsilon)}{\partial \log g}\right)^{2} \sigma_{\text {logg }}^{2}+\left(\frac{\partial \log (\epsilon)}{\partial \xi}\right)^{2} \sigma_{\xi}^{2}}
$$

Given the small uncertainties in stellar parameters, the total errors on $\mathrm{Li}$ and $\mathrm{Al}$ abundances are almost entirely related to the best-fit determination; typical values range from $0.10-0.13$ for $\mathrm{Li}$ and $0.14-0.16$ for $[\mathrm{Al} / \mathrm{Fe}]$.

\section{RESULTS AND DISCUSSION}

Elemental abundances ( $\mathrm{Li}$ and $[\mathrm{Al} / \mathrm{Fe}]$ ) are displayed in Tables 2 and 3, where we list the identification number for each star and $V$ magnitudes (from the Momany et al. photometry), the $\mathrm{S} / \mathrm{N}$ ratios at $6700 \AA$ along with stellar atmospheric parameters (the complete Tables are made available online only). Our results are presented separately for each target cluster in Sections 3.1 and 3.2 for NGC 6218 and NGC 5904, respectively. We then provide a general discussion on the $\mathrm{Li}$ abundance pattern observed in GCs by summarising and discussing findings from this study along with previous investigations (Section 3.3.

\subsection{NGC 6218 (M12)}



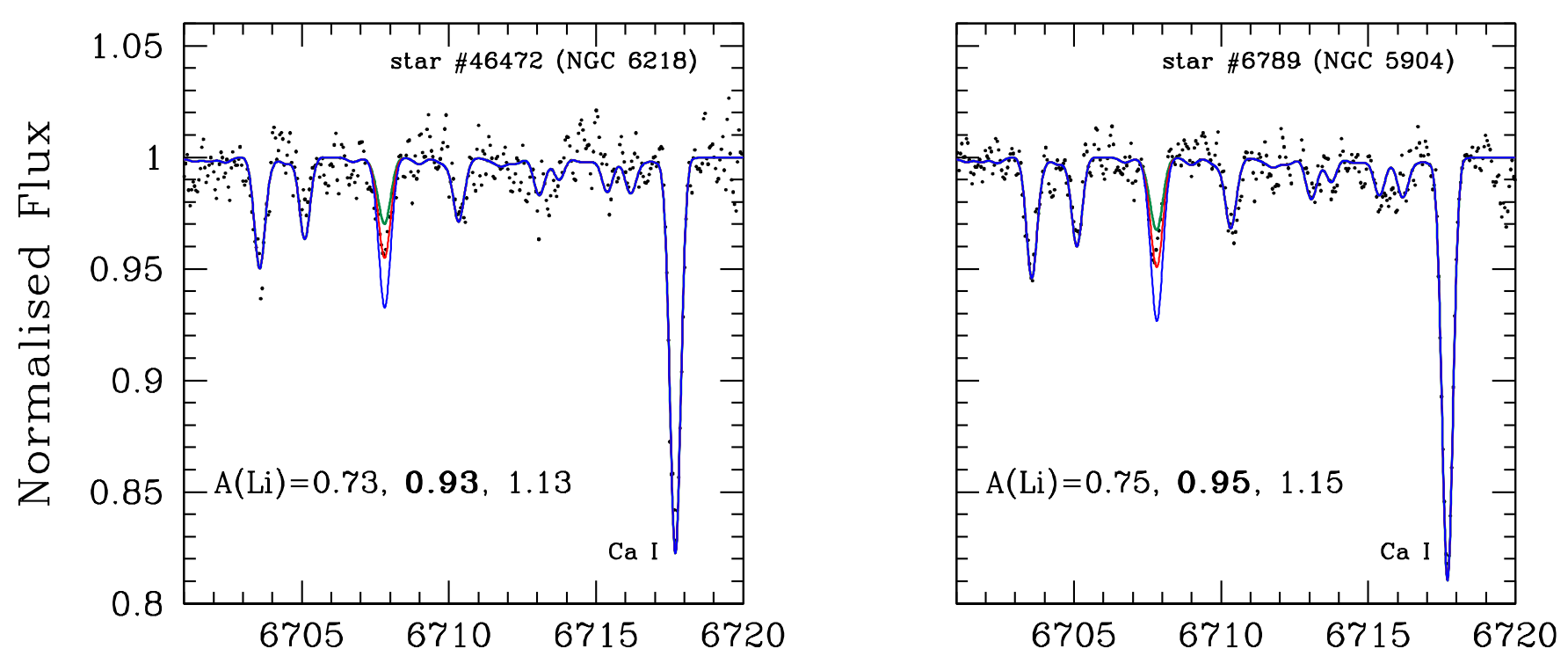

Wavelength $(\AA)$

Figure 2. Example of the spectral synthesis for the Li I line for stars \#46472 (NGC 6218) and \#6789 (NGC 5904).
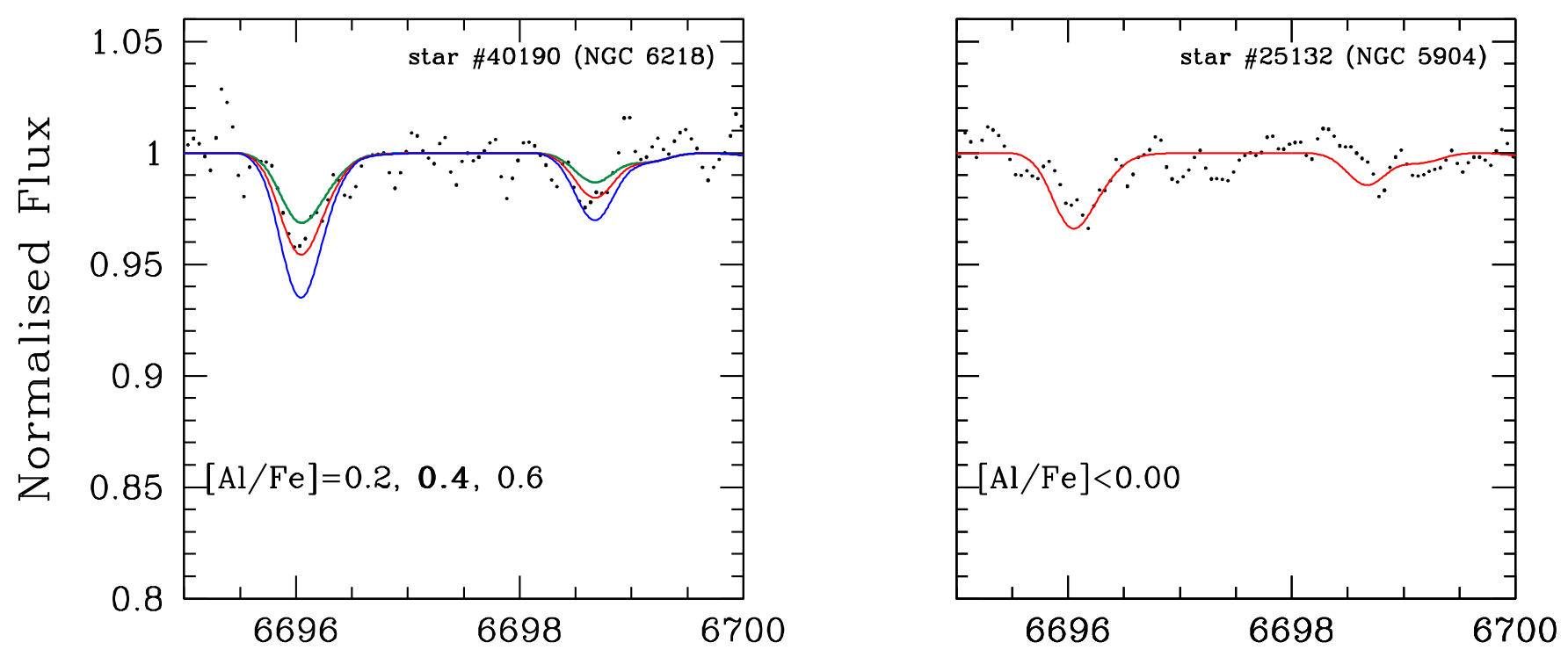

\section{Wavelength $(\AA)$}

Figure 3. Example of the spectral synthesis for the Al I lines for stars \#40190 (NGC 6218) and \#25132 (NGC 5904; here only an upper limit is given).

In Figure 4, $\mathrm{A}(\mathrm{Li})$ is plotted as a function of $V$ magnitude for all our sample stars in NGC 6218. Here blue circles denote detections, black triangles upper limits and the solid grey line indicates the magnitude of the LF bump, located at $V=14.78$ according to Nataf et al. (2013). The magnitude of the LF bump is generally believed to denote the beginning of extra mixing in these low-mass stars: there is excellent agreement between photometry and spectroscopy regarding the onset of this event, with stars exhibiting a declining trend of $\mathrm{Li}$ abundances as a function of luminosity once the bump is reached. The brightest stars in our sample have had their lithium content significantly depleted and only upper limits can be provided. Moreover, a slightly decreasing trend of $\mathrm{Li}$ with magnitudes is present for our targets with $\mathrm{V}$ ranging between $\mathrm{V}=15.75$ and the bump: the correlation coefficient is found to be $r=0.55$, which is significant at more than $99.9 \%$ level (the probability that this happened by chance is less than $0.1 \%$ ). The slope of the correlation is $0.13+/-0.03$, which is very close to the accuracy imposed by our observational uncertainties $(\sim 0.10-0.13$ dex $)$. Given that our errors are 
Table 2

Stellar parameters, $\mathrm{Li}$ and $\mathrm{Al}$ abundances for targets in NGC 6218 (M12). This table is available in its entirety in a machine-readable form in the online journal. A portion is shown here for guidance regarding its form and content.

\begin{tabular}{cccccccc}
\hline \hline Star ID & $V$ & $\mathrm{~S} / \mathrm{N}$ & $T_{\text {eff }}$ & $\log g$ & $\xi$ & $\mathrm{A}(\mathrm{Li})$ \\
& $(\mathrm{mag})$ & & $(\mathrm{K})$ & $(\mathrm{Al} / \mathrm{Fe}]$ \\
& $\left(\mathrm{cm} \mathrm{s}^{-2}\right)$ & $\left(\mathrm{km} \mathrm{s}^{-1}\right)$ & $\mathrm{dex}$ & $\mathrm{dex}$ \\
\hline 10219 & 15.28 & 78 & 4946 & 2.59 & 1.39 & 1.08 & - \\
26629 & 15.60 & 60 & 5016 & 2.75 & 1.34 & 1.06 & 0.60 \\
26778 & 14.57 & 115 & 4787 & 2.23 & 1.50 & 0.53 & 0.00 \\
31393 & 15.17 & 100 & 4921 & 2.53 & 1.41 & 0.93 & - \\
31600 & 14.95 & 90 & 4871 & 2.42 & 1.44 & 0.95 & 0.00 \\
\hline
\end{tabular}

Table 3

Stellar parameters, Li and Al abundances for targets in NGC 5904 (M5). Entries with asterisks indicate upper limits. This table is available in its entirety in a machine-readable form in the online journal. A portion is shown here for guidance regarding its form and content.

\begin{tabular}{lccccccc}
\hline \hline Star ID & $\begin{array}{c}V \\
(\mathrm{mag})\end{array}$ & $\mathrm{S} / \mathrm{N}$ & $\begin{array}{c}T_{\text {eff }} \\
(\mathrm{K})\end{array}$ & $\begin{array}{c}\log g \\
\left(\mathrm{~cm} \mathrm{~s}^{-2}\right)\end{array}$ & $\begin{array}{c}\xi \\
\left(\mathrm{km} \mathrm{s}^{-1}\right)\end{array}$ & $\begin{array}{c}\mathrm{A}(\mathrm{Li}) \\
\mathrm{dex}\end{array}$ & $\begin{array}{c}{[\mathrm{Al} / \mathrm{Fe}]} \\
\mathrm{dex}\end{array}$ \\
\hline 229 & 15.63 & 100 & 5000 & 2.57 & 1.39 & 1.02 & $0.00^{*}$ \\
394 & 15.13 & 130 & 4888 & 2.32 & 1.47 & $0.65^{*}$ & 0.35 \\
1069 & 15.91 & 95 & 5063 & 2.71 & 1.35 & 1.05 & $0.10^{*}$ \\
1476 & 15.03 & 140 & 4865 & 2.27 & 1.49 & 0.97 & $0.00^{*}$ \\
1778 & 15.56 & 115 & 4983 & 2.53 & 1.40 & 1.00 & 0.10 \\
\hline
\end{tabular}

likely overestimated, we are tempted to conclude that this $\mathrm{Li}$ pattern is real. The trend is clearly not related to the multiple population scenario, because it is present in both FG and SG stars (divided according to the $\mathrm{Na}$ and $\mathrm{O}$ abundances, as in Carretta et al. 2009a). As pointed out by the referee, there could be two possible explanations for this trend: $(i)$ in situ depletion as stars evolve along the sub giant branch (contrary to standard theory); or (ii) increased previous Li depletion as a function of mass along the sub giant branch, possibly showing signs of a $\mathrm{Li}$ dip in metal-poor stars analogous to the Population I F dwarf dip.

By considering only giants fainter than the RGB bump, we find a mean $\mathrm{Li}$ abundance of $\mathrm{A}(\mathrm{Li})=0.98 \pm 0.01$ (r.m.s. 0.06, 44 stars). This is consistent with no Li variation in this cluster (the fact that the standard deviation is formally lower than observational uncertainties $-0.10 / 0.13$ dex- indicates that the measurement errors are probably over-estimated). It is implicit that any spread we detect is not the true internal (intrinsic) Li dispersion. There is a component that is due to observational errors. Similarly, in clusters where we state that the dispersion is consistent with no lithium variation, i.e., M4 and M12, there may in fact be a small spread below the measurement uncertainties. We cannot prove zero dispersion, but only provide statistical limits to its size. Given our observational errors, we obtain that the internal dispersion in $\mathrm{Li}$, estimated using the standard deviation from the mean, cannot be larger than about $\sim 0.1$ dex. The constancy of $\mathrm{Li}$ abundances is a noteworthy result because NGC 6218 is known to display, along with the large majority of Galactic GCs, large variations in p-capture elements. As previously mentioned in Section 2 no infor-

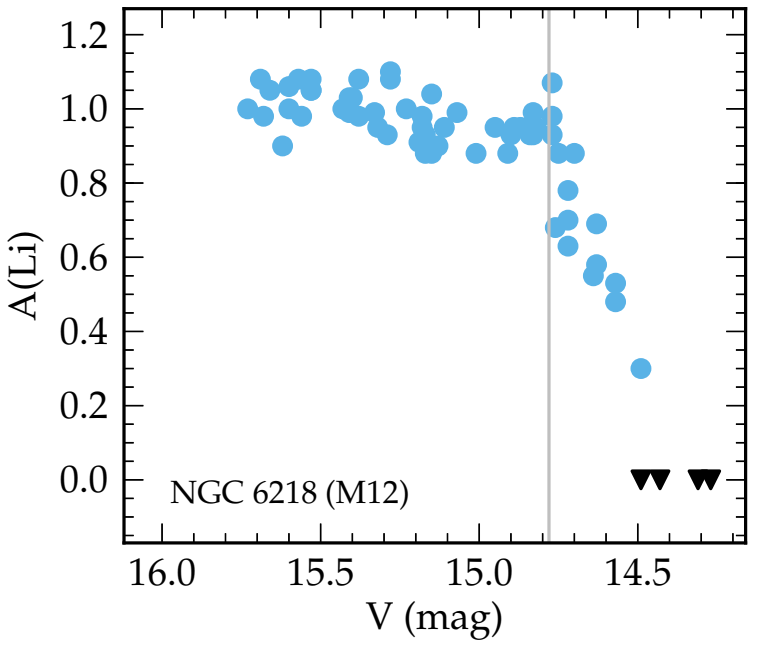

Figure 4. $\mathrm{A}(\mathrm{Li})$ as a function of visual magnitude in the globular cluster NGC 6218 (M12). Blue circles denote lithium detections whereas black triangles represent upper limits. The solid grey line indicates the magnitude of the LF bump in this cluster, $\mathrm{V}=14.78$, as given by Nataf et al. (2013).

mation on $\mathrm{Na}$ and $\mathrm{O}$ abundances can be gathered from our spectra. However, we have stars in common with the survey by Carretta et al. (2007): out of 44 stars (not yet experiencing in situ extra mixing), we have $\mathrm{Na}$ and $\mathrm{O}$ abundances for 21 and 18 stars, respectively. In Figure 5 we show our $\mathrm{A}(\mathrm{Li})$ against their $[\mathrm{Na} / \mathrm{Fe}]$ and $[\mathrm{O} / \mathrm{Fe}]$ ratios for those stars in common: variations of almost 1 dex in $\mathrm{Na}$ and more than 1.2 dex in $\mathrm{O}$ do not coincide with changes in the Li abundance. There seems to be the hint of a weak anticorrelation between $\mathrm{Li}$ and $\mathrm{Na}$ abundances, however the Pearson's correlation coefficient results in $r=-0.38$ (16 degrees of freedom) and is not statistically meaningful: there is a probability larger than $10 \%$ that this correlation could happen by chance. Following the definition introduced by Carretta et al. (2009a), we can group our stars into their respective generation based on their $\mathrm{Na}$ content, with FG stars defined as having $\mathrm{Na}$ abundance of $[\mathrm{Na} / \mathrm{Fe}] \leq[\mathrm{Na} / \mathrm{Fe}]_{\min }+0.3$ dex. We find that FG stars have an average $\mathrm{Li}$ abundance of $\mathrm{A}(\mathrm{Li})$ $=1.00 \pm 0.04$ (r.m.s $=0.09$ ), whilst in $\mathrm{SG}$ stars $\mathrm{A}(\mathrm{Li})$ $=0.98 \pm 0.02$ (r.m.s $=0.06$ ). Thus, the different stellar populations identified according to their $\mathrm{Na}$ abundances are indistinguishable in terms of their $\mathrm{Li}$ content. In Figure 6 we compare the spectra of two stars with very similar parameters $\left(\Delta T_{\text {eff }}=37 K\right)$, but differences in $\mathrm{Na}$ and $\mathrm{O}$ abundances of more than a factor of 2 . It is evident that there is no remarkable difference in the $\mathrm{Li}$ I line strength, entailing that those stars have to share a very similar Li content (virtually the same within the observational uncertainties).

Our spectral coverage also permitted us to obtain $\mathrm{Al}$ abundances for a sub-sample of 54 stars. We derived a mean $\mathrm{Al}$ abundance of $[\mathrm{Al} / \mathrm{Fe}]=+0.21 \pm 0.03$ (r.m.s $=0.19)$, which is in excellent agreement with values published by Carretta et al. (2009b) $([\mathrm{Al} / \mathrm{Fe}]=$ $0.20 \pm 0.05$, r.m.s $=0.18$ ) based on a sample of 11 bright giants observed with the high-resolution UVES spectrograph. Johnson \& Pilachowski (2006) analysed intermediate-resolution spectra $(\mathrm{R} \approx 15000)$ for $21 \mathrm{RGB}$ stars in this GC, deriving stellar parameters, metallicity, 

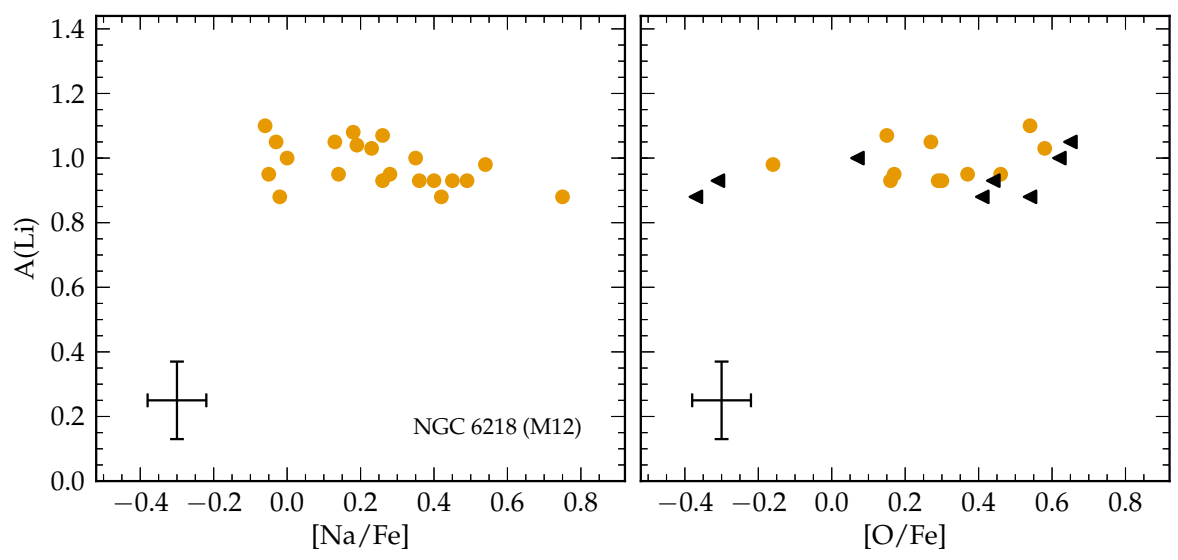

Figure 5. Left Panel: A(Li) for stars below the RGB bump as a function of $[\mathrm{Na} / \mathrm{Fe}]$ and $[\mathrm{O} / \mathrm{Fe}]$ as determined by Carretta et al. (2007). Orange circles denote detections and triangles upper limits in $\mathrm{O}$ abundances. Error bars indicate the typical internal error.

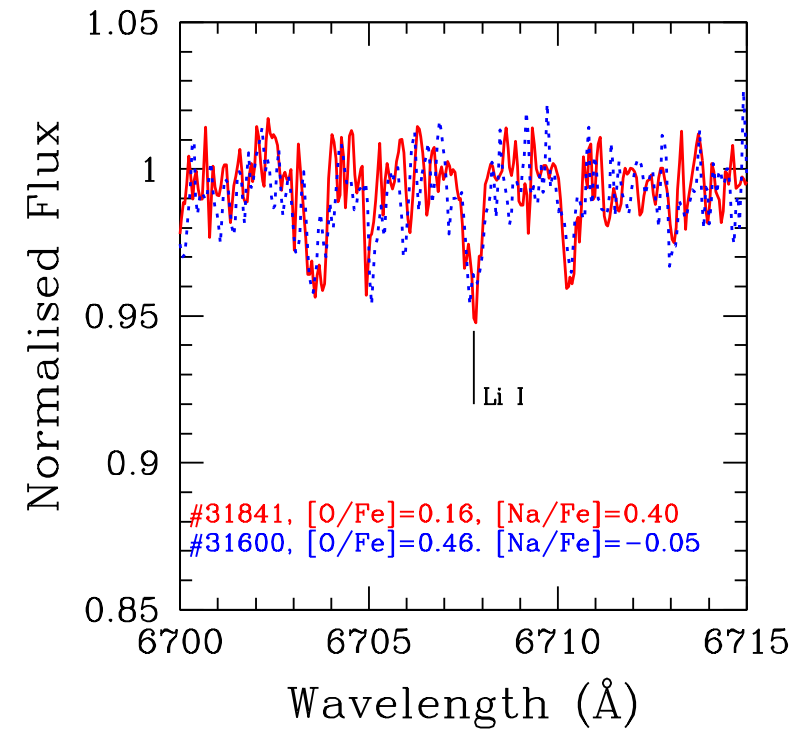

Figure 6. Comparison of the spectra for two stars in M12 with very similar stellar parameters and Li abundances, but very different $\mathrm{Na}$ and $\mathrm{O}$ content (see text for discussion).

p-capture and n-capture element abundances. They obtained an average $[\mathrm{Al} / \mathrm{H}]=-1.00 \pm 0.03$ compared to our value of $[\mathrm{Al} / \mathrm{H}]=-1.16 \pm 0.03$ (we directly compare $[\mathrm{Al} / \mathrm{H}]$ because there is an offset in metallicity of about 0.2 dex between the two studies). Taking into account the measurement uncertainties, and a difference in the $\log g f$ for the $\mathrm{Al}$ doublet at $6696-6698 \AA$ of 0.20 and 0.24 (in the sense ours minus theirs), the two mean values agree very well.

Our results confirm that the $\mathrm{Al}$ content does vary in this $\mathrm{GC}$ and that $\mathrm{Al}$ and $\mathrm{Na}$ abundances are positively correlated, as expected from the activation of $\mathrm{NeNa}$ and $\mathrm{MgAl}$ cycles. This is demonstrated in Figure7, where our $[\mathrm{Al} / \mathrm{Fe}]$ ratios are plotted as a function of $[\mathrm{Na} / \mathrm{Fe}]$ from Carretta et al. for stars in common. Our sample stars span a range of $\approx 0.8 \mathrm{dex}$ in $[\mathrm{Al} / \mathrm{Fe}]$ (peak-to-peak variation), very similar to the value found by Carretta et al. (2009b) from UVES spectra (i.e., $\Delta[\mathrm{Al} / \mathrm{Fe}] \approx 0.7 \mathrm{dex}$ ), whereas this is larger than what has been found by Johnson \& Pilachowski (2006), $\Delta[\mathrm{Al} / \mathrm{Fe}] \approx 0.4$ dex. It is not straightforward to determine the cause of such dis-

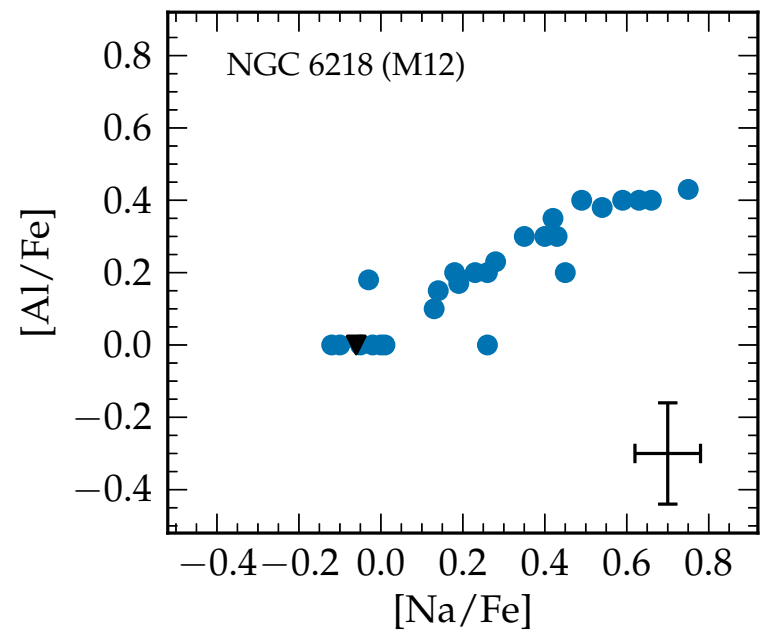

Figure 7. $[\mathrm{Al} / \mathrm{Fe}]$ from the present study as a function of the $[\mathrm{Na} / \mathrm{Fe}]$ derived by Carretta et al. (2007)

crepancy, and statistics may certainly play a role (our sample is a factor of 2 larger). However, we note that according to Johnson \& Pilachowski (2006) there are no Al-poor stars (FG) in their sample. The minimum value in this population being $[\mathrm{Al} / \mathrm{Fe}]=0.35 \mathrm{dex}$ (while the maximum $\mathrm{Al}$ abundance is in good agreement with our value as well as with those by Carretta et al. 2009b, i.e., roughly at $\approx 0.7$ dex level).

In Figure 8 we plot $\mathrm{A}(\mathrm{Li})$ as a function of $[\mathrm{Al} / \mathrm{Fe}]$ abundance, where lavender circles denote detections and the black triangle indicates an upper limit to the $[\mathrm{Al} / \mathrm{Fe}] \mathrm{ra}-$ tio. If we compare the variation in $\mathrm{Al}$ from our entire sample $(\approx 0.8$ dex $)$ to that we determined from common stars with Carretta et al. (2009b, see Figure 7 $\approx 0.4$ dex) there is almost a factor of two difference. This might be a mere statistical effect. However, it could be that, since stars in common between the two works are brighter, the scatter increases as luminosity decreases (because the spectral lines get weaker at higher temperatures). We checked the presence of possible trends between our $[\mathrm{Al} / \mathrm{Fe}]$ ratios and the $V$ magitudes and we concluded that there is a slight increase in the $\mathrm{Al}$ dispersion at lower luminosity but the trend is weak (the effect is anyway well within the observational uncertainties).

Analogously to what is revealed in the $\mathrm{Li}-\mathrm{O}$ and $\mathrm{Li}-$ 


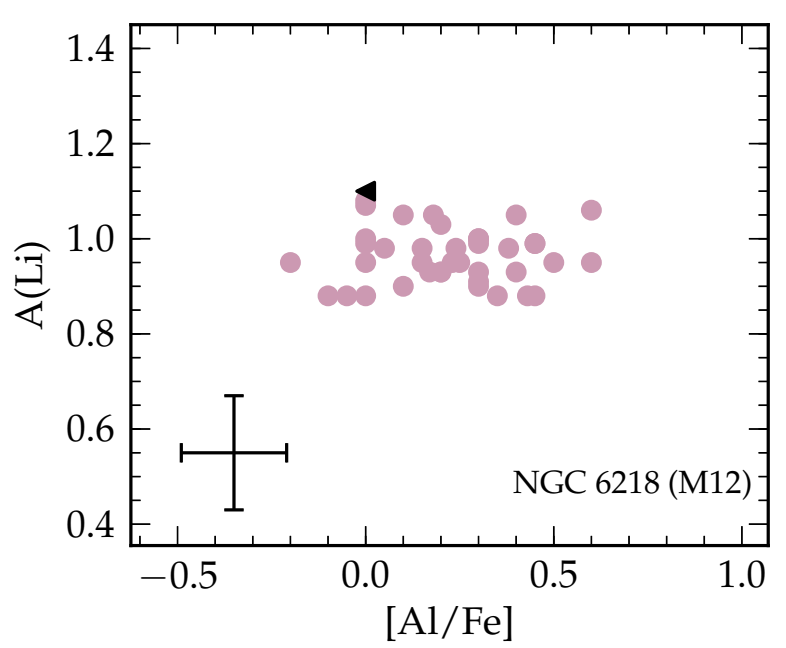

Figure 8. $\mathrm{A}(\mathrm{Li})$ for stars below the RGB bump as a function of $[\mathrm{Al} / \mathrm{Fe}]$ abundance in the globular cluster NGC 6218 (M12). Lavender circles denote lithium detections whereas the black triangle represents an upper limit to the $[\mathrm{Al} / \mathrm{Fe}]$ abundance. The typical internal error for the abundances are indicated by the error bar.

Na planes, while Al displays a large variation among our sample stars, the $\mathrm{Li}$ abundance remains constant. The large variations in all the p-capture elements under scrutiny here are not accompanied by analogous changes in $\mathrm{Li}$. The implication is that $\mathrm{Li}$ production must have occurred across the different stellar generations, ruling out a major contribution by FRMS to the GC internal enrichment, and favouring the IM-AGB candidate. In this regard, M12 is "M4-like" in its behaviour; we recall that both clusters are of similar (current) mass and metallicity (see Section 3.3 for further discussion).

\subsection{NGC 5904 (M5)}

We obtained Li abundances for 99 stars in the massive GC NGC $5904(\mathrm{M} 5,[\mathrm{Fe} / \mathrm{H}]=-1.29$ dex); once again the magnitude range of our sample included giants beyond the LF bump. Our derived Li abundances are shown as a function of the $V$ magnitudes in Figure 9. with symbols retaining their meaning from Figure 4 (i.e., blue circles denote detections, black triangles denote upper limits and the solid grey line indicates the magnitude of the LF bump). This GC possesses two features worthy of mention. First, spectroscopically it is ambiguous as to what magnitude extra mixing begins in this cluster. The photometrically derived LF bump, it could be argued, is located at a $V$ magnitude beyond which stars have already begun to deplete their lithium: compared to the trend observed in Figure 4 the onset of extra mixing is not as clear as in M12. Despite the fact that our photometric system is different from that of Nataf et al. (2013), we determined very similar values for the location of the LF bump. Nataf et al. (2013) derived $V_{\text {bump }}=14.96 \pm 0.01$ compared to $V_{\text {bump }}=14.97 \pm 0.04$ from the present work. Thus, we can state that no major systematic offsets are present between the two catalogues. On the other hand, a small shift to fainter magnitudes of $\approx 0.1 \mathrm{mag}$ for the bump luminosity would result in an agreement between the photometric and spectroscopic determined location at which extra mixing begins. Such a small difference in the required magnitude could

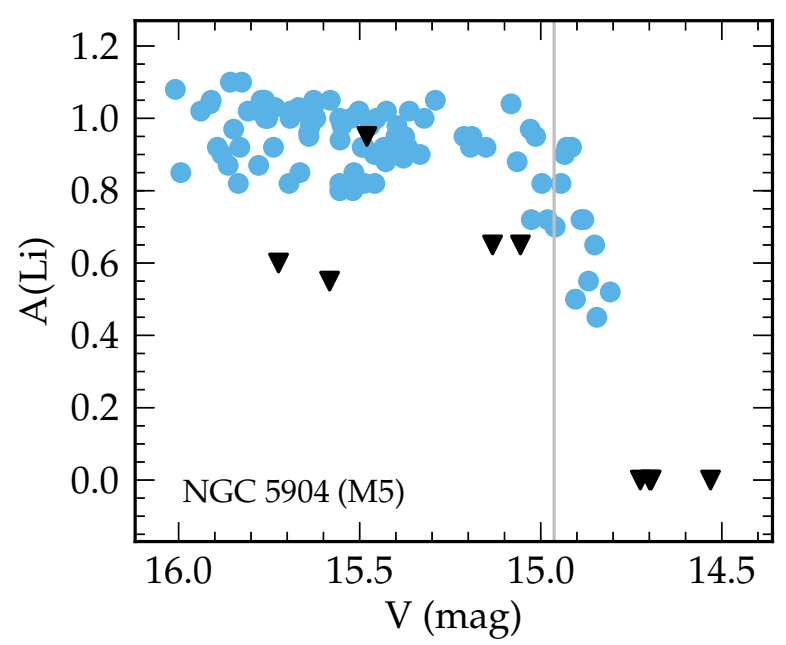

Figure 9. $\mathrm{A}(\mathrm{Li})$ as a function of visual magnitude in the globular cluster NGC 5904 (M5). Symbols are as for Figure 4 The solid grey line indicates the magnitude of the LF bump at $\mathrm{V}=14.96$ as given by Nataf et al. (2013).

be attributed to observational errors. Nevertheless, it will be a point of caution in discussions hereinafter. Secondly and most interestingly, two stars in particular (the two upper limits with $\mathrm{V}$ magnitude $>15.5$ in Figure 9) were found to be severely lithium deficient for their evolutionary phase. Both their radial velocity measurements and metallicity suggest that they are indeed members of the cluster but their Li abundances are inconsistent with the post-FDU composition of the other stars. Whether this translates to deeper FDU, some sort of extra mixing, a rare evolutionary event (although it had to happen at least twice) or it is related to variations in p-capture elements remains unclear (see the following discussion).

When considering the 82 stars that are below the magnitude of the LF bump, we find a mean lithium abundance of $\mathrm{A}(\mathrm{Li})=0.93 \pm 0.01$ (r.m.s 0.11). The standard deviation is roughly of the same order of magnitude of the observational uncertainties, however we need to bear in mind that in the computation of the average, the upper limits in $\mathrm{A}(\mathrm{Li})$ are treated as detections. This indicates that the r.m.s is certainly a lower limit to the actual internal dispersion in Li abundances. Furthermore, as already stated in Section 3.1, the measurement errors are quite likely overestimated. Unfortunately, we have only 16 and 11 stars for which Carretta et al. (2009a) have gathered $\mathrm{Na}$ and $\mathrm{O}$ abundances; in Figure 10 we show the run of $\mathrm{Li}$ with $[\mathrm{Na} / \mathrm{Fe}]$ and $[\mathrm{O} / \mathrm{Fe}]$ ratios for stars in common between the two spectroscopic investigations. There is no evidence for a $\mathrm{Li}-\mathrm{O}$ positive correlation nor for a $\mathrm{Li}-\mathrm{Na}$ anticorrelation: $\mathrm{Na}$ and $\mathrm{O}$ extend for $\approx 0.7$ dex, whilst the Li remain almost constant. The only previous determination of $\mathrm{Li}$ abundance in this GC is that by Lai et al. (2011) who derived Li abundances for three RGB stars below the RGB bump and found an average of $\mathrm{A}(\mathrm{Li})$ $=0.81 \pm 0.06$ (r.m.s $=0.11$ ). The authors concluded that, given the small size of their sample, they can not comment on the relationship between $\mathrm{Li}$ and p-capture elements (e.g., C, Na, O). The exiguous number of common stars between this work and Carretta et al. (2009a) may, however, have prevented us from unveiling the presence of $\mathrm{Li}$ variations in conjunction with the other species 

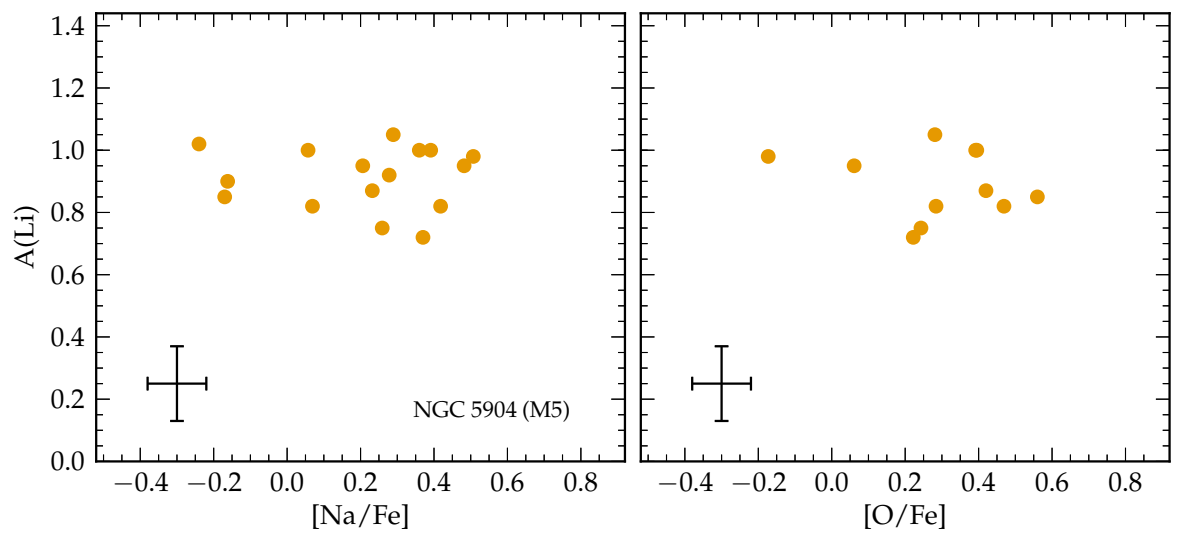

Figure 10. Left Panel: $\mathrm{A}(\mathrm{Li})$ for stars below the RGB bump as a function of $[\mathrm{Na} / \mathrm{Fe}]$ as determined by Carretta et al. (2009a). Right Panel: $\mathrm{A}(\mathrm{Li})$ from this study as a function of $[\mathrm{O} / \mathrm{Fe}]$ as determined by Carretta et al. (2009a). Orange circles denote stars for which the abundances of both species of interest have been measured and error bars indicate the typical internal error in each ratio.

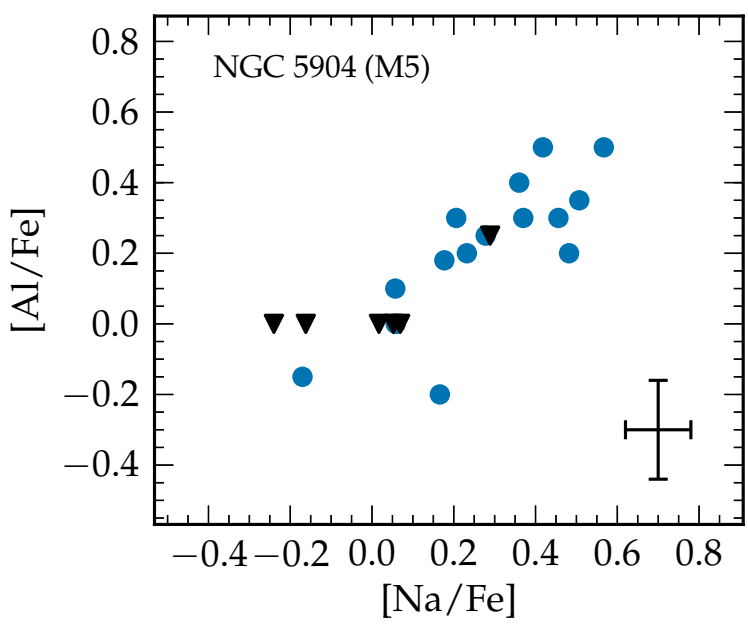

Figure 11. $[\mathrm{Al} / \mathrm{Fe}]$ from the present study as a function of the $[\mathrm{Na} / \mathrm{Fe}]$ derived by Carretta et al. (2009a)

involved in the hot H-burning, such as $\mathrm{Na}$ and $\mathrm{O}$. Thus, to get deeper insights into the possible relationship between $\mathrm{Li}$ and p-capture elements, we derived the $\mathrm{Al}$ abundances for a total of 93 stars. From our sample we detected a peak-to-peak variation in the $[\mathrm{Al} / \mathrm{Fe}]$ ratio of $\approx 0.7$ dex, which is the same value found by Ivans et al. $(2001)$ and is consistent with Shetrone $(1996, \approx 0.6)$ and Carretta et al. (2009b, $\approx 0.8 \mathrm{dex})$. Considering the subgroup of stars in common with Carretta et al. (2009a, 21 stars), we report our derived $\mathrm{Al}$ abundances as a function of the Na determined by the Carretta et al study in Figure 11. We obtained a very clear $\mathrm{Na}-\mathrm{Al}$ correlation, with a Pearson's correlation coefficient $r=0.81$, which is significant at more than $99.99 \%$. In this respect, we confirm results from previous studies (see Carretta et al. 2009b, Ivans et al. 2001, Shetrone 1996).

As was previously done for NGC 6218, we include a discussion on the $[\mathrm{Al} / \mathrm{Fe}]$ and $\mathrm{Li}$ abundances for those stars in the cluster yet to begin in situ extra mixing. By adopting a formal $V_{\text {bump }}$ of 14.96 , as given from photometry (but keeping in mind possible mismatches between photometry and spectroscopy, as mentioned at the beginning of this Section), we plot the derived $\mathrm{A}(\mathrm{Li})$ abundances as a function of $[\mathrm{Al} / \mathrm{Fe}]$ in Figure 12. Here laven-

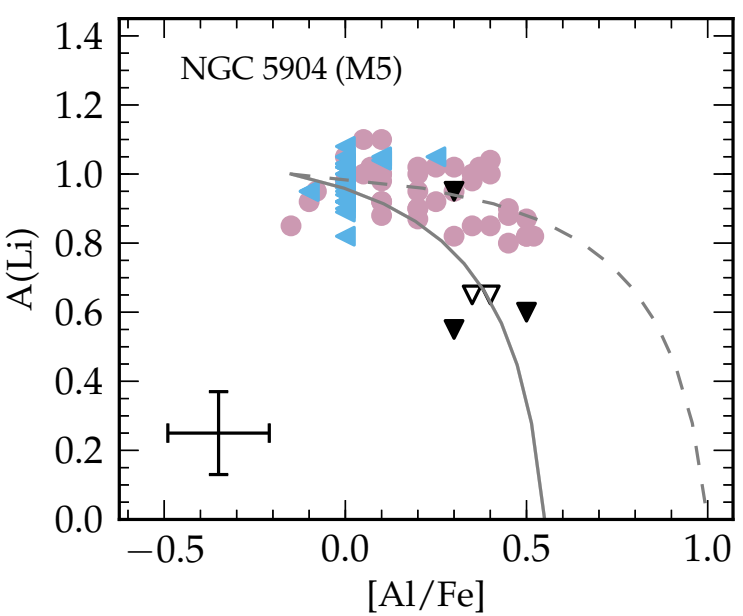

Figure 12. $\mathrm{A}(\mathrm{Li})$ for stars below the RGB bump as a function of $[\mathrm{Al} / \mathrm{Fe}]$ abundance in the globular cluster NGC 5904 (M5). Lavender circles denote measurements for both species, black triangles denote upper limits to the lithium abundance and blue triangles denote upper limits to the $[\mathrm{Al} / \mathrm{Fe}]$ abundance. The two "peculiar" stars close to the bump are marked as empty (black) triangles. The typical internal errors are indicated. The curves represent different dilution models (see text for discussion).

der circles denote stars where both $\mathrm{Li}$ and $\mathrm{Al}$ have been measured, black triangles represent stars for which an upper limit to the lithium abundance has been determined and blue triangles represent upper limits in the derived $[\mathrm{Al} / \mathrm{Fe}]$ abundance. There is evidence for a $\mathrm{Li}-\mathrm{Al}$ anticorrelation, with a Pearson's coefficient $\mathrm{r}=-0.44$ (75 degrees of freedom) which is significant at more than $99.9 \%$ (the probability that this event can happen by chance is lower than $0.1 \%$ ). If we discard those two stars for which the extra mixing might already have begun (i.e., stars very close to the bump and labelled as empty triangles here to emphasise their different behaviour), the anticorrelation is still present $(\mathrm{r}=-0.40)$. Note that even had we discarded the other two upper limits $(V>15.5)$, there is still evidence for anticorrelation between $\mathrm{Li}$ and $\mathrm{Al}$ abundances $(r=-0.33$, significance level at $99.9 \%)$ However, these other two stars that exhibit Li depletion have magnitudes $V>15.5$, so they are much fainter than the bump. They demonstrate the expected abundance 


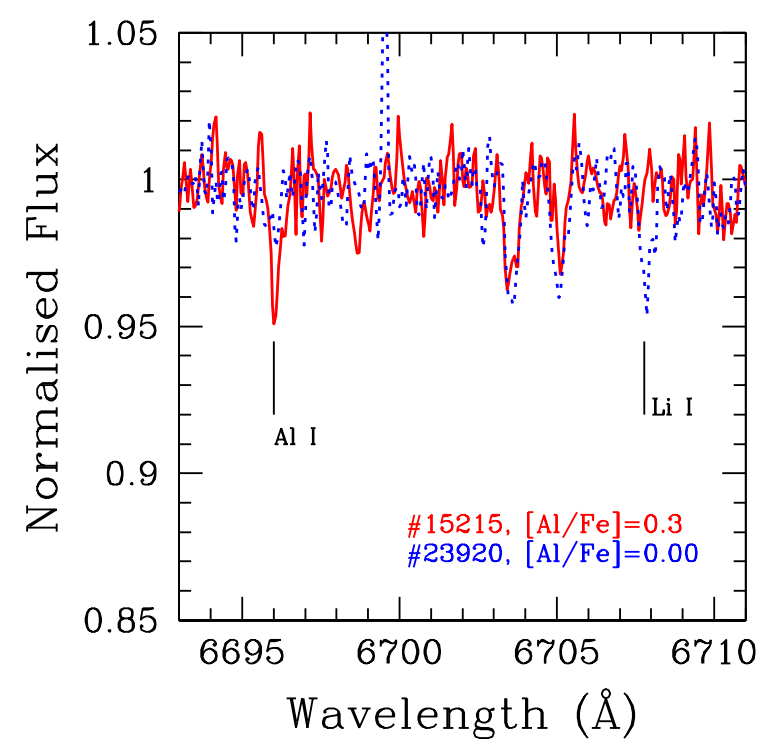

Figure 13. Comparison of the spectra for two stars in M5 with different $\mathrm{Li}$ and $\mathrm{Al}$ abundances (see text for discussion).

pattern if we assume that the processed material that forms the SG stars is Li-poor and Al-rich. To convince the reader of this possibility, we show in Figure 13 the spectral comparison for one of these stars, namely star \#15215, with another GC member with identical atmospheric parameters (star \#23920, $\Delta T_{\text {eff }}=9 \mathrm{~K}$ ). As is clear from the Figure, \#15215 is Li-poor and (relatively) Alrich, whereas \#23920 is Li-rich and Al-poor. Thus, we might be tempted to conclude that the two (and perhaps the four?) stars that exhibit significant Li depletion (and corresponding $\mathrm{Al}$ enhancement) constitute the extreme (E) SG stars in M5. However, having detected so few of these stars would entail that the fraction of $\mathrm{E}$ stars we obtain is about the $3 \pm 1 \%$ of the cluster population, which is lower than the value of $7 \pm 2 \%$ found by Carretta et al. (2009a) according to their $\mathrm{Na}$ and $\mathrm{O}$ abundances (see that paper for details on the definition of the PIE groups).

In order to gain a better understanding of the chemical abundance pattern emerging from this study, we determined a dilution model for this GC, as per Prantzos \& Charbonnel (2006). In this model $[X]$, the logarithmic abundance of species $\mathrm{X}$, is a mixture (given by a dilution factor, $d$ ) of the original abundance, $\left[X_{O}\right]$, and processed material, $\left[X_{p}\right] .[X]$ is determined such that:

$$
[X]=\log \left[(1-\mathrm{d}) 10^{\left[\mathrm{X}_{\mathrm{o}}\right]}+\mathrm{d} \times 10^{\left[\mathrm{X}_{\mathrm{p}}\right]}\right] .
$$

In Figure 12 we plot as solid line the dilution model with initial abundances of $\mathrm{Li}=1.00$ and $[\mathrm{Al} / \mathrm{Fe}]=-0.15$ and processed material having $\mathrm{Li}=0.00$ and $[\mathrm{Al} / \mathrm{Fe}]=0.55$, based on the extrema measured in our sample. As can be easily seen from the plot, this dilution curve fails in reproducing the observed trend. More specifically, we can identify three groups of stars: (1) stars that show primordial $\mathrm{Li}$ and $\mathrm{Al}$ abundances (FG stars); (2) stars with primordial Li but enhancement in their Al content, at different levels; (3) stars with an extreme composition, characterised by paucity of $\mathrm{Li}$ and increased $\mathrm{Al}$ abundances (SG stars, with an extreme pattern). The majority of the GC stars, that belong to group 2 , cannot be explained by diluting the primordial population with the extreme SG, because they still exhibit a quite large Li abundance (the curve is indeed a lower envelope to their distribution). This implies that in order to reproduce their $\mathrm{Li}$ abundance, we have to call for a $\mathrm{Li}$ production within the stellar polluters.

Alternatively, one possible solution requires the presence of an unobserved population, typyfied by $[\mathrm{Al} / \mathrm{Fe}]$ of approximately $\approx 1.0$ dex (the dashed curve in Figure 12). Both this survey and Carretta et al. (2009b) have failed to identify potential candidates. There might be two reasons why this population is unseen:

- There are no stars formed from the pure ejecta (i.e., with a pollution fraction of $100 \%$ ). The processed material coming from IM-AGB stars would in this case have $\mathrm{Al}$ enhancements of more than $[\mathrm{Al} / \mathrm{Fe}] \approx 1$ $\operatorname{dex}$ (and is naturally $\mathrm{Na} / \mathrm{N} / \mathrm{He}$ rich also). This material is required to mix with primordial material (and hence become diluted) before the formation of the SG started. In a recent paper, D'Antona et al. (2012) examined dynamical models where it is possible, in principle, to accumulate and mix the ejecta for a time $\mathrm{t}_{f}$ before starting star formation (see Table 1 of their paper). However, there is no obvious explanation as to the cause of the delay in the star formation events. Such a scenario requires that the gas from the AGB stars is collected at the GC centre and remains in a quiescent condition for $\approx$ 40-50 Myr. Star formation is inhibited until the cleared pristine material (swept out from the SN II explosions) can fall back and mix to produce the the subsequent stellar generation.

- These very peculiar stars, characterised by extreme Al over-abundances, should also possess a huge amount of He. At a given age, stars with larger amount of $\mathrm{He}$ are less massive than their counterpart with normal He (i.e., Y 0.24). Considering the metallicity of M5, stars with $Y>0.35$ will have $\mathrm{M} \lesssim 0.5 \mathrm{M}_{\odot}$ ( see Gratton et al. 2010a; 2010b) and they might not reach the RGB tip. In fact, Castellani \& Castellani (1993) have shown that if the mass is smaller than that required to activate the He-core flash, the stars will become $R G B$ manqué. In this circumstance, the He-flash can occur at high effective temperatures after stars have left the RGB (the so-called "hot flashers") and they eventually move to the blue hook of the horizontal branch (HB, see e.g., Moehler et al. 2002). Unfortunately, as widely discussed in Gratton et al. (2013), we can not determine the chemical composition for stars warmer than the Grundahl u-jump (Grundahl et al. 1999), because of severe sedimentation and radiative levitation effects.

Nevertheless, if this is the case, then an explanation for the two (or even four) Li-poor stars in Figure 12 would still be required, perhaps calling for a rare event of extra mixing that begins well before the LF bump luminos- 
ity is reached 11 At the moment there is no compelling evidence to reject them, only because no satisfactory explanation for the cluster's dilution history has presented itself. In summary, NGC 5904 displays a degree of complexity that can not be accounted for by a simple dilution model. This cluster demonstrates the presence of three different stellar populations. This is reminiscent of what Carretta et al. (2012) discovered in the GC NGC 6752, where the intermediate SG stars cannot be explained by simply considering a mixture of primordial composition and (extreme) highly-processed material (i.e., the E stars).

\subsection{The Li spreads in GCs}

Our investigation into the $\mathrm{Li}$ abundance and its potential spread within M12 and M5 has revealed that these GCs behave differently. In M12 we recover a chemical pattern very similar to that previously observed in M4 by DM10 (and corroborated by other authors, see e.g., Mucciarelli et al. 2011; Villanova \& Geisler 2011). Because FG and SG stars share exactly the same Li abundance, while showing depletion in $\mathrm{O}$ of more than $50 \%$, we require that the GC polluters have contributed ashes enriched in $\mathrm{Li}$. As a consequence, FRMS (and massive binaries) cannot be responsible for this trend, because the current theory suggests that they carry Li-free ejecta. On the other hand, in M5 we disclosed the presence of a rather peculiar and complex chemical composition: a simple dilution model fails in reproducing the three populations currently co-existing in the cluster. Furthermore, we also need to invoke Li production within the polluters to explain the abundance pattern in the majority of the GC stars (i.e., the SG stars which are Al-rich but still Lirich). Crucially, we detected the presence of an extreme population, which is characterised by $\mathrm{Al}$ overabundances and Li deficiency (as expected in the case of hot $\mathrm{H}$ burning). These stars were not revealed in our M12 sample; however statistics might have played a role in this respect: given that we have 54 stars for which $\mathrm{Li}$ and $\mathrm{Al}$ have been measured, assuming that the fraction of extreme stars is as in M5 (i.e., about 3\%) we would expect to find at least one of those stars. The probability that we missed all of them is $19 \%$, which is not negligible. This should be regarded as a point of caution in the following discussion.

These two GCs share a very similar metallicity, but they significantly differ in current mass. M5 is much more massive than M12 (and M4). In order to determine what role GC mass plays in the Li distribution among the different stellar populations, we plot in Figure 14 the internal spread in $\mathrm{Li}, \Delta \mathrm{A}(\mathrm{Li})$, as a function of the absolute visual magnitude (a proxy for the current cluster mass). We used, as a proxy for the Li distribution, the peak-to-peak variation within each GC. This obviously includes the contribution related to the observational uncertainties, implying that the intrinsic Li spread could be

\footnotetext{
11 It is also possible that these stars are binaries; we might have captured post blue straggler stars (BSS) in our RGB sample, which could explain the Li depletion (Rvan et al. 2002). As already mentioned, the radial velocity is consistent with the cluster mean, but multi-epoch observations to detect possible variations are currently not in hand. We note, however, that the fact that all of them are Al-rich seems to suggest that the Li deficiency is somehow related to the multiple population scenario.
}

smaller than the peak-to-peak values (and virtually zero in cases like M4/12). To evaluate the extent of the $\mathrm{Li}$ spreads in our target GCs, we considered only the stars with magnitudes fainter than the RGB bump luminosity. The peak-to-peak variation in the $\mathrm{Li}$ abundances is 0.22 dex and 0.55 dex for NGC 6218 and NGC 5904, respectively. Had we eliminated the four upper limits in M5 the peak-to-peak variation would be 0.40 dex. As there was a non-negligible probability that we missed corresponding extreme stars in M12, both values for the $\mathrm{Li}$ spread in M5 are plotted in Figure 14. Regardless of which value we adopt, the observed Li spread in M5 is larger than in M12 (recall our observational uncertainties are the same for the two clusters). We included data for the GC NGC 6397 by selecting a subsample of stars analysed by Lind et al. (2009): in order to be as homogeneous as possible with our target giants, we restricted our attention to those RGB stars within approximately 1 magnitude fainter than the bump. This choice, although limiting the sample size, allows us to minimise the impact of the star-to-star difference in the atmospheric parameters (especially in temperatures, which can increase the internal scatter) and guarantees a reliable comparison with our GC giants. We determined a Li spread of 0.18 dex. We proceeded in the same fashion by including Li abundances for a subsample of giants published by Mucciarelli et al. (2011). We find the $\mathrm{Li}$ variation to be 0.25 dex. Unfortunately for the GCs 47 Tuc and NGC $6752, \mathrm{Li}$ abundances in the giant stars have not been determined and we are forced to exploit dwarfs. This should be a point of caution when considering the general trend shown in Figure 14. Note, in the same context, that the spectra for NGC6752 by Shen et al. (2010) are characterised by very low $\mathrm{S} / \mathrm{N}$ ratios, in same cases below 15 , possibly suggesting that the spread quoted is overestimated because of the observational uncertainties.

We detect, for the first time to our knowledge, the existence of an unambiguous correlation between the $\mathrm{Li}$ variation and the total cluster luminosity (i.e., mass; the Pearson's correlation coefficient $\mathrm{r}=-0.93$ is significant at more than 99.9\%): the more massive the $G C$, the larger the Li spread12. This finding seems to suggest that $\mathrm{Li}$ production is less efficient in the more massive GCs than in small GCs, because any kind of Li replenishment tends to erase the presence of $\mathrm{Li}-\mathrm{O}$ and/or $\mathrm{Li}-\mathrm{Na}$ anticorrelation. We find that in less massive systems the FG and SG stars are very similar as far as the Li content is concerned (even indistinguishable in some cases like M4 and M12). We can speculate that in less massive GCs, the polluter mass range might be biased towards the lower end of the IM-AGB stars $\left(\mathrm{M} \lesssim 6 \mathrm{M}_{\odot}\right)$ whilst in the most massive GCs we expect the upper envelope of the mass distribution to extend beyond $\left(\mathrm{M} \gtrsim 7 \mathrm{M}_{\odot}\right)$. This is required to account for the considerable p-capture element variations observed in these massive GCs, such as e.g., NGC 2808, where high levels of $\mathrm{O}$ depletion and $\mathrm{Na}$ enhancement $([\mathrm{O} / \mathrm{Fe}]$ down to $\approx-1.00$ and $[\mathrm{Na} / \mathrm{Fe}]$ up to $\approx+1.00)$, as well as significant $\mathrm{Mg}$ depletion and Al enrichment have been reported. Regarding Li, any comparison between observed chemical abundances and

\footnotetext{
12 Note that the correlation is still significant at more than $95 \%$ level if we adopt for $\mathrm{M} 5$ the value of $\Delta \mathrm{A}(\mathrm{Li})=0.40$, that results from ignoring the four upper limits for the extreme population
} 
AGB models must be done bearing in mind all the uncertainties involved. Li production is indeed extremely sensitive to the input physics in the stellar models: in the IM-AGBs there is a very brief phase of $\mathrm{Li}$ enrichment at the stellar surface during the first few thermal pulses, with a peak of $\mathrm{A}(\mathrm{Li}) \sim 4$ dex, but the final $\mathrm{Li}$ yield depends on when the star loses most of its mass (Ventura \& D'Antona 2010; D'Orazi et al. 2013). The rate and details of the mass loss are among the most uncertain (and difficult to model) factors in theoretical stellar astrophysics.

Interestingly, we have demonstrated that the FG and SG stars display the same $\mathrm{Li}$ abundances in GCs like M4 and M12. These results imply that the internal polluters must have produced $\mathrm{Li}$ in roughly the same amount as the primordial abundances. A $5 \mathrm{M}_{\odot}$ AGB stellar model published in D'Orazi et al. (2013) results in $\mathrm{A}(\mathrm{Li})=2.00$, by adopting an increased $\alpha_{\mathrm{MLT}}=2.2$ and Bloecker (1995) mass loss law, and $\mathrm{A}(\mathrm{Li})=2.35$ with a "standard" $\alpha_{\mathrm{MLT}}=1.75$ and Bloecker mass loss (see Figure 20 of that paper). Similar Li yields have been found previously by Ventura \& D'Antona 2010. If we assume that standard Big Bang nucleosynthesis is correct, then FG stars have formed with an initial Li content of $\mathrm{A}(\mathrm{Li}) \sim 2.7-2.8$ and they subsequently depleted their $\mathrm{Li}$ abundances by a factor of $\approx 3$ to the Spite plateau value. Unless SG stars somehow deplete $\mathrm{Li}$ in a different way compared to FG, they also should have born with $\mathrm{A}(\mathrm{Li}) \sim 2.7-2.8$, implying that the polluters must be capable of producing such high Li abundance. Although D'Orazi et al. (2013) found the Li production being lower for AGB models of 5 and $6 \mathrm{M}_{\odot}$, we recall that those yields are highly dependent on mass loss rate. By adopting an even more extreme mass loss than Bloecker, we can reach a higher Li production. On the other hand, there is still the possibility that standard Big Bang nucleosynthesis is not correct, and that both FG and SG stars formed with Li abundances of $\mathrm{A}(\mathrm{Li}) \sim 2.1-2.3$ dex, with no need to invoke $\mathrm{Li}$ depletion in both stellar generations. Were this the case, then theoretical models for AGB stars would result in fair agreement with observational measurements without further adjustments to the input physics. Although there is much evidence to support the predictions of standard Big-Bang Nucleosynthesis (Steigman 2007), we must give consideration to these possibilities.

The clusters reported in Figure 14 are obviously characterised by a range in metallicity. The sample includes the metal-poor GC NGC $6397([\mathrm{Fe} / \mathrm{H}] \approx-2.0$ dex $),$ NGC $6752([\mathrm{Fe} / \mathrm{H}] \approx-1.5 \mathrm{dex})$, the intermediatemetallicity GCs M12, M5 and M4 $([\mathrm{Fe} / \mathrm{H}] \approx-1.3 \mathrm{dex})$ and one of the most metal-rich GCs in 47 Tuc $([\mathrm{Fe} / \mathrm{H}]$ $\approx-0.7 \mathrm{dex})$. Given the limited sample size available, we cannot robustly infer the level of contribution provided by metallicity on the spread in $\mathrm{Li}$ in GCs. We note that two GCs with almost the same mass (M4 and NGC 6752), but slightly dissimilar $[\mathrm{Fe} / \mathrm{H}]$, do actually exhibit $\mathrm{Li}$ variations at a different extent. Irrespective of possible metallicity-related effects, the trend appearing in Figure 14 points to the GC mass as the driving parameter of the anticorrelation. This is evident when we compare GCs with similar metallicity but different masses (e.g., M5 vs M12 and M4). It is noteworthy, in this context, that these GCs also display different HB morphology, M4 and M5 being the classical exam-

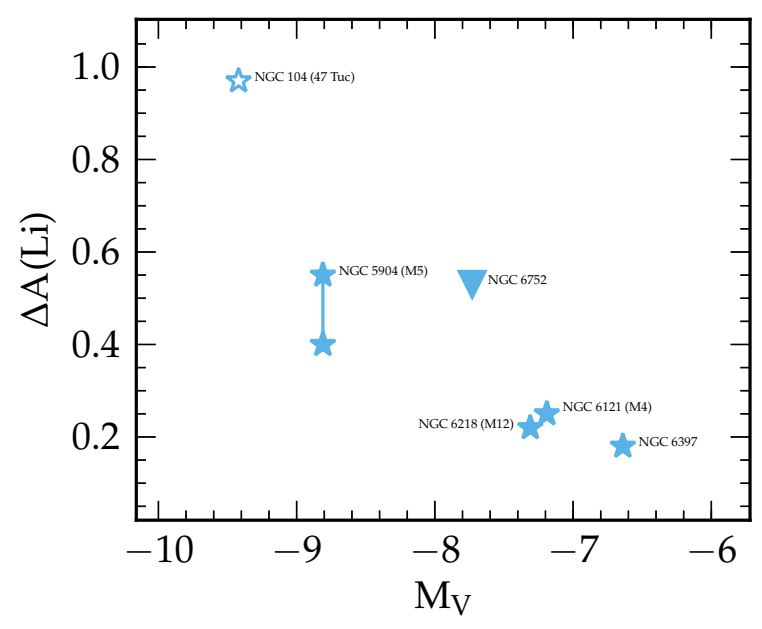

Figure 14. $\Delta \mathrm{A}(\mathrm{Li})$ (peak-to-peak variation) as a function of $M_{\mathrm{V}}$. For further details about the computation of these values see text.

ple of the so-called "second-parameter" pair (see e.g., Gratton et al. 2013 and references therein).

The metal-rich GC 47 Tuc (NGC 104, labelled with a different symbol in Figure 14) deserves further discussion. In fact, in this $\mathrm{GC}$ the large scatter detected in the $\mathrm{Li}$ abundance seems to be completely unrelated to variations in p-capture elements and, more generally unrelated to the presence of multiple stellar populations. As discussed in D'Orazi et al. (2010b), while SG (Opoor) stars are characterised by a low Li abundance, there is a huge spread within the first stellar generation itself, $\mathrm{A}(\mathrm{Li})$ ranging from $\sim 1.5$ to $\sim 2.5$ (we ignore for the current purposes the presence of a very Li-rich star with $\mathrm{A}(\mathrm{Li})=2.78)$. They concluded that this primordial scatter is probably related to the high metallicity of this GC and is likely the Population II analogue of what is observed in Population I stars with similar atmospheric parameters (such as e.g., in the open cluster M67, Randich et al. 2000). In addition, 47 Tuc is known to display peculiar behaviour also in terms of light-element variations and their relationship with the cluster properties, namely its $\mathrm{Na}-\mathrm{O}$ anticorrelation is relatively short despite the large GC mass. It stands as an outlier in the diagrams of $\mathrm{IQR}^{[3]}[\mathrm{Na} / \mathrm{O}]$ vs $\mathrm{M}_{V}$ and $\log T_{\text {eff,max }}^{\mathrm{HB}}$ (the maximum temperature on the $\mathrm{HB}$ ), as derived by Carretta et al. (2010). By discarding this GC, we still can detect the hint for an anticorrelation but the small statistics prevents us from conclusively confirming its existence. The situation will become clear once a larger sample of GCs (encompassing a wide range in current mass) becomes available in the present survey.

\subsubsection{The Origin of the Li Spread}

The aim of the work presented here has been to constrain the nature of the progenitor stars that are most responsible for the abundance patterns observed in GCs. The determination of $\mathrm{Li}$ abundances in GC stars provide a means to differentiate between those progenitors that are predicted to produce $\mathrm{Li}$ and those that are predicted only to destroy it. GCs such as M4 and M12

\footnotetext{
13 Interquartile range
} 
show very little (if any) difference in the lithium abundance between the two populations, requiring significant Li production in the progenitors (to account for the high Li content still present in SG stars). M5 also seems to require some lithium production across the different stellar generations, but not to the same extent. Furthermore, we have identified a possible correlation between the current mass of the GC and the degree of Li spread. We have proposed that this Li variation is related to the amount of Li produced in the progenitors, however there is another contribution we must consider.

Standard stellar models are unable to produce the observed dispersion in $\mathrm{Li}$ unless there is some form of enrichment. In some instances, particularly when the dispersion is small, the effects of rotation and the instabilities associated with angular momentum loss may be responsible for the dispersion and might in principle affect our ability to put tight constraints on the mass range of the polluting progenitors. Models by Pinsonneault et al. (1992) and discussed in Delivannis et al. (1993) have demonstrated that Li depletion during the pre MS and MS is dependent on the stellar structure (mass, composition, age) and rotational properties (initial angular momentum, timescale for decay). Models calculated with rotation successfully reproduce the large $\mathrm{Li}$ dispersion observed on the MS of open clusters and the small dispersion observed in old metal-poor halo stars. Their Population II models, with a representative distribution in mass, metallicity and angular momentum found in the field significantly deplete Li within the first Gyr of the stellar lifetimes. Models that deplete lithium by up to 1 dex produce a dispersion of 0.1 dex and those that deplete Li by $0.3-0.5$ dex result in a dispersion of $0.03-0.05$ dex.

In the context of the multiple populations in GCs, such a small Li dispersion (at about $\sim 0.1$ dex level) may be entirely due to the distribution in angular momentum of second generation stars. It would imply all FG stars formed from a similar angular momentum distribution as those in the halo. Whilst SG stars in more massive clusters form with a larger range of initial angular momenta leading to a larger range in lithium depletion. Thus, although Li production within the polluters seems to be mostly responsible for the total internal spread in $\mathrm{Li}$ abundances, a further (minor) contribution due to e.g., MS depletion cannot be discarded. In fact, observational data for M4 and M12 (e.g., Mucciarelli et al. 2011 or this study) are in agreement with a very limited Li variation (within $0.1 \mathrm{dex}$ ), which might be explained in terms of phenomena unrelated to the multiple populations frameworks, such as e.g., MS depletion due to rotational-induced mixing.

\section{SUMMARY AND CONCLUDING REMARKS}

Lithium provides rare insight into not only the internal processes of stars but also the internal chemical enrichment of GCs. The abundance patterns within these old stellar aggregates are most straightforwardly explained by the presence of multiple populations, whereby a first generation of stars has polluted the medium from which a second generation form. Within this second generation it is still possible for distinct chemical populations to form (see Carretta et al. 2009a and their PIE definitions). Because of its fragility and thus the special conditions re- quired for its production, Li may serve as a unique tracer of the nature of stars that provided the intra-cluster enrichment. Current stellar theory predicts that it is possible for intermediate-mass asymptotic giant branch stars to produce lithium via the Cameron-Fowler mechanism, whereas fast-rotating massive stars and massive binaries will produce Li-free ejecta. How lithium correlates with other p-capture species will reveal whether this element has been produced between the different stellar generations and thus help to identify the progenitors. Following on from the work of DM10 who focussed on NGC 6121, we have presented lithium and $[\mathrm{Al} / \mathrm{Fe}]$ abundances in stars on the RGB of the GCs NGC 6218 and NGC 5904. Our findings can be summarised as follows:

In the GC NGC 6218 (M12), any Li variation is less than observational errors and is consistent with no $\mathrm{Li}$ variation between the two populations. Thus, whilst the cluster displays clear $[\mathrm{Na} / \mathrm{Fe}]$ (over 1 dex) and $[\mathrm{O} / \mathrm{Fe}]$ (over $1.2 \mathrm{dex}$ ) variations, stars across their respective populations remain indistinguishable according to their $\mathrm{Li}$ abundance. These variations in $\mathrm{Na}$ and $\mathrm{O}$ are accompanied by variations in the $[\mathrm{Al} / \mathrm{Fe}]$ abundance. The (anti)correlations that form between p-capture nuclei are expected when hydrogen burning at high temperatures has been in operation ( $\mathrm{T} \gtrsim 20 \mathrm{MK})$. Because the large variations in p-capture elements are not accompanied by corresponding changes in $\mathrm{Li}$, Li production must have occurred across the different stellar generations. With our current understanding of stellar evolution, such a result favours a major contribution from IM-AGB progenitors, as also found by DM10 in the analogous GC NGC 6121 (M4).

In the GC NGC 5904 (M5), we are unable to statistically confirm $\mathrm{Li}$ variation with $\mathrm{O}$ or $\mathrm{Na}$; however there is a hint for a $\mathrm{Li}-\mathrm{Al}$ anticorrelation. We anticipate that the small number of stars with both $\mathrm{Li}$ and $\mathrm{Na}$ (or $\mathrm{O}$ ) measured is hindering our ability to detect a relationship between these species. There are possibly four (two confirmed) stars that are very lithium poor for their evolutionary phase. They may be due to non-standard evolution or perhaps members of an extreme (third) population in this cluster, that displays Li deficiency and $\mathrm{Al}$ enhancement. Given the presence of a $\mathrm{Li}$ - $\mathrm{Al}$ anticorrelation in NGC 5904, we have fit a dilution model as per Prantzos \& Charbonnel (2006) to explain the chemical history of the cluster. When we mix the composition representative of the primordial population with that of four candidate extreme members, we are unable to account for the abundances of a majority of the cluster stars (which are Li-rich and Al-rich). To do so would require the pristine material to be combined with an unobserved population that has $[\mathrm{Al} / \mathrm{Fe}]$ of approximately $\approx 1.0$ dex. If this is the case, by trying to reproduce a majority of the cluster members we still require an explanation for the (four) extreme population candidates. Thus, NGC 5904 exhibits a level of complexity much higher than its more standard siblings, like M4 and M12. This result is not surprising when we consider the HB morphology of this GC.

There is a clear anticorrelation between the internal Li spread and the current GC mass. Although Pinsonneault et al. (1990) and Pinsonneault et al. (1992) have shown that rotation during the MS can produce dispersions in the $\mathrm{Li}$ abundance, we attribute the 
spread to production in the progenitors. Li production appears less efficient in the more massive GCs than in smaller GCs, pointing to a different mass range of the stellar polluters involved in the internal enrichment. Further surveys will help constrain this indication on more robust grounds and reveal what role metallicity plays in the lithium spread found within GCs.

This work made extensive use of the SIMBAD, Vizier, and NASA ADS databases. We thank Simon Campbell, Maria Lugaro, and Enrico Vesperini for very helpful discussions. VD is an ARC Super Science Fellow. EC, RGG, SL, and YM acknowledge partial support by PRIN INAF 2011 "Multiple populations in globular clusters: their role in the Galaxy assembly" (PI E. Carretta). We thank the referee for a very careful reading of the manuscript and for her/his very helpful comments and suggestions

\section{REFERENCES}

Alonso, A., Arribas, S., \& Martínez-Roger, C. 1999, A\&AS, 140, 261

Anderson, J., Piotto, G., King, I. R., Bedin, L. R., \&

Guhathakurta, P. 2009, ApJ, 697, L58

Angelou, G. C., Church, R. P., Stancliffe, R. J., Lattanzio, J. C., \& Smith, G. H. 2011, ApJ, 728, 79

Angelou, G. C., Stancliffe, R. J., Church, R. P., Lattanzio, J. C., \& Smith, G. H. 2012, ApJ, 749, 128

Bastian, N., Lamers, H. J. G. L. M., de Mink, S. E., Longmore, S. N., Goodwin, S. P., \& Gieles, M. 2013, MNRAS, 436, 2398

Bedin, L. R., Piotto, G., Anderson, J., Cassisi, S., King, I. R., Momany, Y., \& Carraro, G. 2004, ApJ, 605, L125

Bloecker, T. 1995, A\&A, 297, 727

Bonifacio, P., et al. 2007, A\&A, 470, 153

Bragaglia, A., Gratton, R. G., Carretta, E., D’Orazi, V., Sneden, C., \& Lucatello, S. 2012, A\&A, 548, A122

Cameron, A. G. W., \& Fowler, W. A. 1971, ApJ, 164, 111

Cardelli, J. A., Clayton, G. C., \& Mathis, J. S. 1989, ApJ, 345, 245

Carretta, E., Bragaglia, A., Gratton, R., \& Lucatello, S. 2009b, A\&A, 505, 139

Carretta, E., Bragaglia, A., Gratton, R. G., Leone, F., Recio-Blanco, A., \& Lucatello, S. 2006, A\&A, 450, 523

Carretta, E., Bragaglia, A., Gratton, R. G., Lucatello, S., \& D'Orazi, V. 2012, ApJ, 750, L14

Carretta, E., Bragaglia, A., Gratton, R. G., Recio-Blanco, A., Lucatello, S., D'Orazi, V., \& Cassisi, S. 2010, A\&A, 516, A55

Carretta, E., Gratton, R. G., Bragaglia, A., D’Orazi, V., \& Lucatello, S. 2013, A\&A, 550, A34

Carretta, E., Lucatello, S., Gratton, R. G., Bragaglia, A., \& D'Orazi, V. 2011, A\&A, 533, A69

Carretta, E., et al. 2007, A\&A, 464, 939

- 2009a, A\&A, 505, 117

Castellani, M., \& Castellani, V. 1993, ApJ, 407, 649

Castelli, F., \& Kurucz, R. L. 2004, ArXiv Astrophysics e-prints

Cottrell, P. L., \& Da Costa, G. S. 1981, ApJ, 245, L79

Cyburt, R. H., Fields, B. D., \& Olive, K. A. 2008, J. Cosmology Astropart. Phys., 11, 12

D'Antona, F., D'Ercole, A., Carini, R., Vesperini, E., \& Ventura, P. 2012, MNRAS, 426, 1710

de Mink, S. E., Pols, O. R., Langer, N., \& Izzard, R. G. 2009 , A\&A, 507, L1

de Silva, G. M., Gibson, B. K., Lattanzio, J., \& Asplund, M. 2009, A\&A, 500, L25

Decressin, T., Meynet, G., Charbonnel, C., Prantzos, N., \& Ekström, S. 2007, A\&A, 464, 1029

Deliyannis, C. P., Pinsonneault, M. H., \& Duncan, D. K. 1993, ApJ, 414, 740

Denissenkov, P. A., \& VandenBerg, D. A. 2003, ApJ, 593, 509

D’Orazi, V., Campbell, S. W., Lugaro, M., Lattanzio, J. C., Pignatari, M., \& Carretta, E. 2013, MNRAS, 433, 366
D’Orazi, V., Gratton, R., Lucatello, S., Carretta, E., Bragaglia, A., \& Marino, A. F. 2010a, ApJ, 719, L213

D'Orazi, V., Lucatello, S., Gratton, R., Bragaglia, A., Carretta, E., Shen, Z., \& Zaggia, S. 2010b, ApJ, 713, L1

D’Orazi, V., \& Marino, A. F. 2010, ApJ, 716, L166

González Hernández, J. I., et al. 2009, A\&A, 505, L13

Gratton, R., Sneden, C., \& Carretta, E. 2004, ARA\&A, 42, 385

Gratton, R. G., Carretta, E., \& Bragaglia, A. 2012, A\&A Rev., 20,50

Gratton, R. G., Carretta, E., Bragaglia, A., Lucatello, S., \&

D’Orazi, V. 2010a, A\&A, 517, A81

Gratton, R. G., Carretta, E., \& Castelli, F. 1996, A\&A, 314, 191

Gratton, R. G., D’Orazi, V., Bragaglia, A., Carretta, E., \&

Lucatello, S. 2010b, A\&A, 522, A77

Gratton, R. G., Sneden, C., Carretta, E., \& Bragaglia, A. 2000,

A\&A, 354, 169

Gratton, R. G., et al. 2001, A\&A, 369, 87

-. 2013, A\&A, 549, A41

Grundahl, F., Catelan, M., Landsman, W. B., Stetson, P. B., \& Andersen, M. I. 1999, ApJ, 524, 242

Harris, W. E. 1996, AJ, 112, 1487

Ivans, I. I., Kraft, R. P., Sneden, C., Smith, G. H., Rich, R. M., \& Shetrone, M. 2001, AJ, 122, 1438

James, G., François, P., Bonifacio, P., Carretta, E., Gratton,

R. G., \& Spite, F. 2004, A\&A, 427, 825

Johnson, C. I., \& Pilachowski, C. A. 2006, AJ, 132, 2346

- 2010, ApJ, 722, 1373

Kraft, R. P. 1994, PASP, 106, 553

Kurucz, R. 1993, ATLAS9 Stellar Atmosphere Programs and 2

km/s grid. Kurucz CD-ROM No. 13. Cambridge, Mass.:

Smithsonian Astrophysical Observatory, 1993., 13

Lai, D. K., Smith, G. H., Bolte, M., Johnson, J. A., Lucatello, S. Kraft, R. P., \& Sneden, C. 2011, AJ, 141, 62

Lind, K., Primas, F., Charbonnel, C., Grundahl, F., \& Asplund, M. 2009, A\&A, 503, 545

Marino, A. F., Villanova, S., Piotto, G., Milone, A. P., Momany, Y., Bedin, L. R., \& Medling, A. M. 2008, A\&A, 490, 625

Marino, A. F., et al. 2011, ApJ, 731, 64

Milone, A. P., Marino, A. F., Piotto, G., Bedin, L. R., Anderson, J., Aparicio, A., Cassisi, S., \& Rich, R. M. 2012, ApJ, 745, 27

Moehler, S., Sweigart, A. V., Landsman, W. B., \& Dreizler, S. 2002, A\&A, 395, 37

Momany, Y., Bedin, L. R., Cassisi, S., Piotto, G., Ortolani, S., Recio-Blanco, A., De Angeli, F., \& Castelli, F. 2004, A\&A, 420, 605

Momany, Y., Cassisi, S., Piotto, G., Bedin, L. R., Ortolani, S., Castelli, F., \& Recio-Blanco, A. 2003, A\&A, 407, 303

Mucciarelli, A., Salaris, M., \& Bonifacio, P. 2012, MNRAS, 419, 2195

Mucciarelli, A., Salaris, M., Lovisi, L., Ferraro, F. R., Lanzoni, B., Lucatello, S., \& Gratton, R. G. 2011, MNRAS, 412, 81

Nataf, D. M., Gould, A. P., Pinsonneault, M. H., \& Udalski, A. 2013, ApJ, 766, 77

Pasquini, L., Bonifacio, P., Molaro, P., Francois, P., Spite, F., Gratton, R. G., Carretta, E., \& Wolff, B. 2005, A\&A, 441, 549

Pasquini, L., et al. 2002, The Messenger, 110, 1

Pinsonneault, M. H., Deliyannis, C. P., \& Demarque, P. 1992, ApJS, 78, 179

Pinsonneault, M. H., Kawaler, S. D., \& Demarque, P. 1990, ApJS, 74, 501

Piotto, G., et al. 2012, ApJ, 760, 39

Prantzos, N., \& Charbonnel, C. 2006, A\&A, 458, 135

Ramírez, S. V., \& Cohen, J. G. 2002, AJ, 123, 3277

Randich, S., Pasquini, L., \& Pallavicini, R. 2000, A\&A, 356, L25

Richard, O., Michaud, G., \& Richer, J. 2005, ApJ, 619, 538

Ryan, S. G., Gregory, S. G., Kolb, U., Beers, T. C., \& Kajino, T. 2002, ApJ, 571, 501

Salaris, M., Weiss, A., Ferguson, J. W., \& Fusilier, D. J. 2006, ApJ, 645, 1131

Shen, Z.-X., Bonifacio, P., Pasquini, L., \& Zaggia, S. 2010, A\&A, 524, L2

Shetrone, M. D. 1996, AJ, 112, 1517

Skrutskie, M. F., et al. 2006, AJ, 131, 1163

Smith, G. H. 2008, PASP, 120, 952

Sneden, C. A. 1973, PhD thesis, THE UNIVERSITY OF TEXAS AT AUSTIN.

Spite, F., \& Spite, M. 1982, A\&A, 115, 357 
Steigman, G. 2007, Annual Review of Nuclear and Particle

Villanova, S., \& Geisler, D. 2011, A\&A, 535, A31

Science, 57, 463

Ventura, P., \& D'Antona, F. 2010, MNRAS, 402, L72

Ventura, P., D'Antona, F., Mazzitelli, I., \& Gratton, R. 2001, ApJ, 550, L65 